\title{
XXVI.
}

\section{Die Indices auctorum und die wirklichen Quellen der Naturalis historia des Plinius.}

\author{
(Quellenstudien zur Nat. hist. Teil II.)
}

In meiner Inaugural-Dissertation glaube ich den Nachweis geführt zu haben, daß̧ die über die 36 Bücher der Naturgeschichte verstreuten Zeitangaben nach Jahren der Stadt, obgleich Plinius selbst varronisch rechnet und eine Tabelle varronischer Aera benutzt, überwiegend capitolinisch datiert erscheinen ${ }^{1}$ ). Aus dieser auffallenden Discrepanz wie aus der nicht minder befremdlichen Erscheinung, daß wir uns, soweit ich beobachtet habe, überall in der Nat. hist., wo für die Zeit des Verfassers nicht passende Zeitangaben begegnen, auf Schriftquellen der augustisch-tiberischen Zeit hingewiesen sehen, an die sich Plinius gedankenlos angelehnt hat ${ }^{2}$ ), habe ich den Schluf gezogen: der Hautstrom der unmittelbaren Quellen der Nat. hist. gehört nicht, wie es dieherrschende Ansichtist, der Zeit der römischen Republik, sondern der ersten Kaiserzeit an. Nun ist das Ergebnis meiner Dissertation, insbesondere unsere auf die Lesart der jedesmal besten Handschrift sich gründende Festlegung der den Daten zu Grunde liegenden Aeren von Münzer und Detlefsen unter Hinweis auf die Unsicherheit der Ueberlieferung in Zweifel gezogen worden ${ }^{3}$ ). Bevor ich es daher unternehme, die

1) Quellenstudien zur Naturalis historia des Plin. Teil I. Die Zeitangaben varronischer und capitolinischer Aera in der Nat. hist. Berl. Diss. 1905 E. Ebering.

2) Vgl. die Uebersicht in m. Diss. S. $49 \mathrm{ff}$.

3) Wochenschr. f. class. Phil. 1905 Sp. 978 . Berl. philol. Wochenschr. $1905 \mathrm{Sp} .1273$. 
Hauptquellen der einzelnen Bücher namentlich zu bestimmen, möchte ich der chronologischen eine andere das Gesamtwerk der Naturgeschichte umfassende Untersuchung an die Seite stellen, die ebensowohl geeignet ist, meine Ansicht von der Abfassungszeit der Hauptquellen in vollem Umfang $\mathrm{zu}$ bestätigen, wie von der Notwendigkeit der liünftig zu befolgenden quellenkritischen Methode zu überzeugen: eine Kritik der Plinianischen Quellenlisten, in denen wir gewohnt sind, eine der vornehmsten Handhaben, wenn nicht die Basis der Quellenforschung zu erblicken, nebst einem von neuen Gesichtspunkten geleiteten Ausblick auf Zeit und Tendenz der von Plinius wirklich benutzten Quellen.

1.

Ueber die Indices auctorum. die neben dem Dedicationsschreiben an Titus und den Indices rerum den Inhalt des ersten Buches der Nat. hist. ausmachen, spricht sich Plinius in der Vorrede folgendermassen aus: $\S 21$ argumentum huius stomachi mei habebis, quod in his voluminibus auctorum nomina praetexui. Est enim benignum, ut arbitror, et plenum ingenui pudoris fateri per quos profeceris, non ut plerique ex his, quos attigi, fecerunt. (22) Scito enim conferentem auctores me deprehendisse a iuratissimis et proximis veteres transscriptos ad verbum neque nominatos, non illa Vergiliana virtute, ut certarent, non Tulliana simplicitate, qui de re publica Platonis se comitem profitetur, in consolatione filiae Crantorem, inquit, sequor, item Panaetium de officiis ... (23) Obnoxii profecto animi et infelicis ingenii est deprehendi in furto malle quam mutuum reddere, cum praesertim sors fiat ex usura.

Für eine unbefangene Interpretation dieses programmatischen Berichts kann es nicht zweifelhaft sein, daß Plinius die Indices nicht in der Absicht, mit seiner Belesenheit zu glänzen, sondern als Ehrenregister für seine Quellen aufgestellt hat, denen dadurch ihr geistiges Eigentum zurückerstattet werden sollte. Insbesondere geht aus den Ausdrücken 'fateri per quos profeceris' und 'mutuum reddere' unzweideutig hervor, dafs unser Autor gewissenhaft alle von ihm wirklich benutzten Quellen und nur diese in den Indices hat aufführen 
wollen. Hieraus mußste der Quellenforschung zunächst die, wie es schien, nicht allzuschwere Aufgabe erwachsen, mit Hilfe der noch erhaltenen Quellen unter den in den Indices genannten Autoren das den ersteren entnommene Material aus dem Text der einzelnen Bücher mit Sicherheit zu eruieren. In Wirklichkeit aber ist es den Gelehrten, die sich darauf beschränkten, die uns erhaltenen Quellen mit dem Gehalt der Nat. hist. zu vergleichen, nicht gelungen, auch nur einen geringen Bruchteil des Materials auf bestimmte Quellen zurückzuführen.

Nun hat vor 50 Jahren Heinrich Brunn den Versuch gemacht, das komparative Verfahren seiner Vorgänger nach einer gesicherten Methode zu leiten ${ }^{4}$ ). Von der Beobachtung ausgehend, daß die Quellenlisten die Autoren in der Regel in derselben Reihenfolge aufführen, wie sie im Text citiert erscheinen, glaubte er die wichtige Entdeckung gemacht zu haben: Plinium eodem ordine quo in componendis libris usus est, auctores etiam in indices rettulisse. Da der Gelehrte es nicht unterlassen hatte, diese seine Hypothese, die schon an sich durch ihre Natürlichkeit und Einfachheit - Brunn selbst nennt sie ein simplicissimum inventum - der Quellenforschung sich aufdrängen mußte, durch einen ausführlichen Beweisapparat zu stützen, hegte man allgemein die Erwartung, es werde nun endlich gelingen, die Plinianische Quellenkritik auf eine sichere Basis zu stellen. In Wahrheit aber hat das Brunnsche Gesetz die Quellenforschung so wenig zu fördern vermocht, daß schon bald ein gewisses Miktrauen gegen die Allgemeingültigkeit und Brauchbarkeit desselben sich geltend machen und die Ansicht ausgesprochen werden durfte: 'Untersuchungen über die Quellenbenutzung des Plinius auf Grundlage der Brunnschen Theorien können erst dann möglicherweise bedeutende Resultate erzielen, wenn in größerem Umfange feste Punkte bestimmt sind, an denen Plinius seine uns noch erhaltenen Quellenschriftsteller sicher benutzt hat' (Detlefsen Phil. XXXI 1872 S. 385 f.) $)^{5}$ ).

4) De auctorum indicibus Plinianis disp. isag. Bonn. 1856.

s) Vgl. Furtwängler Fleckeis. Jahrb. Suppl. IX (1877/78) S. $4 \mathrm{ff}$. Peter Wochenschr. f. class. Phil. $1898 \mathrm{Sp}$. $62 \mathrm{f}$. 
Gleichwohl haben die Späteren immer wieder aus Brunns Hypothese als einem heuristischen Hilfsprincip ersten Ranges Kapital zu schlagen versucht. Man hat sogar mittels des Gesetzes jedem einzelnen in den Indices der kunstgeschichtlichen Bücher genannten Autor Textesstellen zuweisen wollen ${ }^{6}$ ). Aber auch besonnenere Gelehrte haben es nicht unterlassen, sich zur Unterstützung anderweitig begründeter Hypothesen womöglich auf das Brunnsche Gesetz zu berufen ${ }^{7}$, so daf die Worte von Münzer, der zum ersten Mal im Zusammenhang auf eine Reihe von Momenten aufmerksam macht, die die Anwendung des Gesetzes erschweren, die zur Zeit mafgebliche Auffassung bezeichnen: 'Wenn andere Kriterien das bestätigen, was die Anwendung jenes Gesetzes lehrt, so haben wir gesicherte Ergebnisse' ${ }^{8}$ ).

Nun hat sich mir in einer Untersuchung der Quellen des Plinius für die historisch wichtigen Nachrichten aus der Zeit der römischen Republik und der ersten Kaiserzeit das Brunnsche Gesetz als völlig unbrauchbar für die Quellenforschung erwiesen, und bin ich bei einer Revision der berühmten Hypothese zu der Erkenntnis gelangt, daf die Theorien des Gelehrten auf unhaltbaren, ebenso willkürlichen wie irrigen Voraussetzungen beruhen.

Brunn war zuerst die Tatsache aufgefallen, dak die Indices die Autoren gewöhnlich in derselben Reihenfolge verzeichnen, wie sie im Text namentlich citiert erscheinen. Diese Beobachtung legte die Vermutung nahe, daßs Plinius seine Autoren in den Indices in derselben Reihenfolge wie im Text habe anordnen wollen und die jetzigen Abweichungen von dieser Regel in der Hauptsache auf eine spätere Redaction des Textes und dadurch bedingte Umstellungen, Nachträge

6) Urlichs, d. Quellenregister zu Pl.' letzten Btichern 1878. Die gleiche Anschauung (daß „auf dem Brunnschen Gesetz der plinianischen Autorenbenutzung jede weitere Forschung fußen muß“) vertritt Oehmichen in seinen Plinianischen Studien (Erlangen 1880. S. 117). Vgl. auch Hafner quaestiunculae Plin. Neuburg 1898.

7) Mommsen Gesch. d. röm. Münzwesens S. 289 A. 14. Rom. Forsch. II 18 A. 41, II 177. - Hirschfeld, zur Camilluslegende, Festschrift f. Ludw. Friedländer 1895 S. 221.

) Beiträge zur Quellenkritik der Naturgeschichte des Plinins, Berl. 1897 S. 128 ff. 132. 
oder Glossen zurückzuführen seien ${ }^{9}$ ). Da Brunn diese Ansicht mit der seiner Zeit allgemein verbreiteten Anschauung von dem unfertigen Zustand der Nat. hist. in Uebereinstimmung wußte ${ }^{10}$ ), konnte es für ihn, wenn er seine Hypothese zu einem Gesetz erhoben wissen wollte, nur darauf ankommen, nachzuweisen, daß die Abweichungen in der Reihenfolge der Registerautoren von der der Textesautoren durch irgend welche Motive einer späteren Redaction erklärt werden könnten ${ }^{11}$ ).

Nehmen wir einmal mit Brunn an, Plinius habe wirklich die Absicht gehabt, die Registerautoren den namentlichen Citaten im Text entsprechend zu ordnen. Steht denn die Annahme der doppelten Redaction so sehr aufer Zweifel, daß sie zur Begründung einer neuen Theorie verwendet werden darf? Gegen die Präsumption der Nichtvollendung der Naturgeschichte hat sich schon vor 10 Jahren Alfred Gercke in seinen Senecastudien ausgesprochen ${ }^{12}$ ). Allgemein verbreitet aber ist auch heute noch die Ansicht einer auf Plinius selbst zurückgehenden 'Ueber- und Umarbeitung des ursprünglichen

จ) De ind. 2: iam ab aliis observata sunt plurima secundae recensionis vel retractationis vestigia. qua in re ita versatus est Plinius, ut non solum emendaret aut immutaret nonnulla, sed etiam tota capita transponeret vel adeo prorsus novo modo libros divideret, praeterea vero ex auctoribus antea neglectis etiam nova multa addidit, quae in excerpendo, commodius intexendi tempus exspectans, saepe ad marginem adnotasse videtur, unde interdum post ipsius mortem ab imperita manu falso loco in continuitatem verborum inserta sunt. Iam si in fine indicum non semel scriptorum nomina posita invenimus, qui reliqui indicis ordinem turbant, dubitari non potest, quin eos ipsos eodem modo indici non suo loco inseruerit, sed in fine adscripserit.

10) Vgl. die Handbücher d. röm. Litteraturgeschichte von TeuffelSchwabe ${ }^{5}$ II 759 f. $\$ 313$ und Schanz ${ }^{2}$ II 2, 374, 377, wo sich das von Brunn 1. c. formulierte Programm recipiert findet.

11) De ind. 2: quae posita magis quam demonstrata, tamen ita mihi videntur esse comparata, ut veri quandam speciem vel per se is inesse nemo sit negaturus, quare quid inde effici possit, iam in singulis libris experiendum.

12) Fleckeis. Jahrb. Suppl. XI (1896) SXI. 104f.: „Nur aus der Hyperkritik moderner Gelehrter ist die heute verbreitete Anschauung entstanden, daß Plinius die Naturkunde unvollendet seinem Neffen zur Herausgabe überlassen habe. Unleugbare Mängel und Versehen haben auch sorgsamere Schriftsteller aller Zeiten sogar in erheblich kleineren Werken begangen“. - Wie wenig der jüngere Plinius mit dem Werke seines Oheims vertraut war, lehrt überdies ein Vergleich zwischen Plin. epist. VIII 20 und n. h. II 209 , epist IX 33 und n. h. IX 26 (Schanz ${ }^{2}$ II 2, 374 A. 5). 
Textes der Nat. hist.' ${ }^{13}$ ). Ich glaube nicht, daß diese Auffassung noch als dem heutigen Stande unserer Textkritik entsprechend bezeichnet werden darf ${ }^{14}$ ). Wir wissen jetzt, dak die Gelehrten des Altertums nicht so systematisch zu arbeiten pflegten wie wir Modernen. Selbst der groke Systematiker

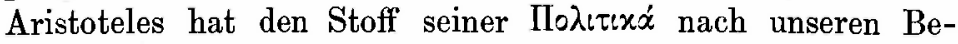
griffen so mangelhaft disponiert, daß die Herausgeber sich vergeblich bemüht haben, in jenes Werk durch Abänderung der überlieferten Bücherfolge und Umstellungen im Text ein geordnetes System hineinzubringen. Sind wir aber heute so weit, daß wir es für unzulässig erklären, die Schwächen und Mängel, die dem Werke eines Aristoteles anhaften, nach formalistischen Principien zu beseitigen ${ }^{15}$ ), so sind wir auch, scheint mir, nicht berechtigt, die Unebenheiten und Nachlässigkeiten in dem Werke eines Kompilators wie Plinius durch spätere Umstellungen, Nachträge oder Interpolationen zu erklären, zumal in einem Kollectaneenwerk eine äuferliche $\mathrm{Zu}$ sammenfügung der Excerpte manchmal kaum zu vermeiden war. Steht also die Annahme einer doppelten Redaction schon an sich auf schwachen Füßen, so können auch noch folgende positive Argumente dagegen geltend gemacht werden. Bekanntlich hat Plinius die Nat. hist. im Jahre 77 seinem Gönner Titus, dem Mitregenten Vespasians (praef. 1 censorius tu sexiesque consul), mit einer Widmung überreicht. Wenn er sein Werk darin mit einem dem kaiserlichen Gaste zu Ehren zugerichteten Festmahl vergleicht und ausdrücklich versichert, ihm, zu dessen Richter er sich Titus ausersehen, die peinlichste Sorgfalt gewidmet zu haben (praef. 6. 8. 11), wenn also unser Autor von der Vorzüglichkeit der ersten Redaction in dieser Weise überzeugt war ${ }^{16}$ ), wird er sich da

19) Detlefsen Herm. XL (1905) S. 575. Vgl. Untersuch. üb. d. Zusammensetz. d. Naturgesch. d. Plin. 1899 S. 18. Münzer Beiträge S. 10. 129. Peter a. a. O. Sp. 64. Teuffel-Schwabe und Schanz a. a. O.

14) Vgl. das Urteil von Gercke a. a. $O$.

15) Vgl. v. Wilamowitz Aristot. und Athen I 187 A. 3 über die , unerträgliche Umstellerei i in den nach der verdienstlichen Edition Bekkers erschienenen Textausgaben der Politik.

${ }^{16}$ ) Der Bezeichnung der Nat. hist. als levioris operae libelli (praef. 12) ist mit Unrecht ein besonderes Gewicht beigelegt worden (RoO archäolog. Aufsätze II 356, Abert die Quellen d. Plin. i. 16. Buche d. N. h. 1896 S. 31. 39, Detlefsen Untersuchungen S. 18). Sollen wir 
in der kurzen Zeit bis zu seinem Tode, während derer er noch durch Amtsgeschäfte als Flottenadmiral in Anspruch genommen war, wirklich zu einer Umarbeitung des Werkes verstanden haben? Was aber vor allem gegen die Annahme einer nachträglichen Redaction spricht: es las sen si ch in der Nat. li ist. keine Tatsachen a uf eisen, die in die beiden letzten Lebensjahre des Plinius fallen ${ }^{17}$ ).

Ferner hat Brunn nicht genug mit der Flüchtigkeit des Plinius in der Herstellung der Indices gerechnet. In $\mathrm{d}$ e $\mathrm{n}$ Listen der B ü cher $3,5-9,14,18,21$ u. a. werden die Namen von Autoren, die in einem einzelnen Paragraphendes Textes genannt werden, in abweichender Reihenfolge aufgeführt. Wie viele von den Abweichungen in der Reihenfolge der in den Registern von der der im Text genannten Autoren werden wir also nicht mit Brunn durch Motive einer späteren Redaction, sondern aus Nachlässigkeiten des Verfassers zu erklären haben!

Diese Bedenken richten sich nicht gegen das Brunnsche Gesetz, sondern gegen die Beweisführung des Gelehrten, die Abweichungen des Textes gegenüber den Quellenverzeichnissen auf eine spätere Bearbeitung der Nat. hist. zurückzuführen. Leider aber ist der ganze Beweisapparat, auch wenn die Annahme einer doppelten Redaction gesichert wäre, nicht einmal geeignet, das von Brunn formulierte Gesetz zu stïtzen. Die Abweichungen, in denen Brunn Störungen erblickte, hätten vielfach als Argumente für die Gültigkeit des Gesetzes verwertet werden können, daßs es nämlich Plinius wirklich darauf

glauben, daß Pl. sich auch im Frnst jedes Talent abspricht, wenn er gleich darauf von seinen libelli sagt: nam nec ingenii sunt capaces quod alioqui nobis perquam mediocre erat? Jene Bezeichnung ist sicher nur eine rhetorische Floskel; denn in den folgenden \$ร kann uns Pl. nicht genug schildern, welche Entsagung ihn seine Arbeit gekostet hat: praef. 14 via nec qua peregrinari animus expetat. nemo apud nos qui idem temptaverit, nemo apud Graecos qui nunc omnia ea tractaverit ...(15) res ardua vetustis novitatem dare .. nobis utique etiam non assecutis voluisse abunde pulchrum. Ueber den rhetorischen Character der Vorrede vgl. auch Schweder Phil. LIV S. 535.

17) Detlefsen Untersuchungen S. 18. Als Jahr der Publication erscheint stets das 830 . d. St. d. i. 77 n. Chr. (n. h. XIV 45, XXVIII 12). 
ankam, seine Quellen in der Reihenfolge, wie er sie benutzt, und nicht blok, wie er sie im Text citiert hatte, in die Register einzutragen.

Bekanntlich haben die rhetorischen Schriftsteller der römischen Kaiserzeit (es sei nur an Velleius, Appian und Dio erinnert) ihre Quellen in der Regel stillschweigend ausgeschrieben, wohl weniger in der Absicht, mit fremdem Eigentum zu glänzen, als mit Rücksicht auf die künstlerische Form der Darstellung, die sie durch Quellencitate nicht beeinträchtigen wollten ${ }^{18}$ ). Auch Plinius konnte nicht umhin, der Vorliebe seines Zeitalters für schöne Form Rechnung zu tragen ${ }^{19}$ ). Im Text hat auch er in der Weise der rhetorischen Schriftsteller seiner Zeit seine Quellen in der Regel stillschweigend ausgezogen. Wenn er sie namentlich citiert, geschieht das nicht in der Absicht, ihnen ihr geistiges Eigentum zurückzuerstatten, sondern aus irgend welchen Motiven der Darstellung, sei es dafi er die Verantwortung für einen Bericht dem genannten Autor zuschieben oder für eine Notiz einen anerkannten Gewährsmann beibringen möchte oder aus anderen Gründen der Art ${ }^{20}$ ). Aber eifert nicht unser Autor in der Vorrede ausdrücklich gegen das bei den Schriftstellern seiner Zeit übliche Verschweigen der unmittelbaren Quellen, und bezeichnet er es nicht gerade als die Pflicht eines anständigen Mannes fateri per quos profeceris und mutuum reddere cum praesertim sors fiat ex usura? Allerdings. An ebenderselben Stelle aber (praef. 21/22 f.) erfahren wir, dab er der ihm

18) Anmerkungen unter dem Text waren bekanntlich dem Altertum fremd.

$\left.{ }^{19}\right)$ Wie sehr ihm die künstlerische Gestaltung seiner sterilis materia am Herzen lag, gesteht er selbst in der Vorrede: $\$ 15$ res ardua vetustis novitatem dare, novis auctoritatem, obsoletis nitorem, obscuris lucem, fastiditis gratiam ... nobis utique etiam non assecutis voluisse abunde pulchrum atque magnificum est. Vgl. n. h. XVIII 214 non auctoribus positis id enim verbosum est.

$\left.{ }^{20}\right)$ Vgl. Gellius noct, Att. praef. 18, Solinus Coll. rer. mem. praef. 6 p. 2, 14 M. ${ }^{2}$, Münzer Beiträge S. $36 \mathrm{ff}$. über die Columellacitate in der N. h., meine Diss. S. 60 A. 26 über das Verriuscitat nat. hist. VIII 17 und Peter a. a. O. S. 68: ,wenn PI. bestimmt citiert, so hat dies in der Regel eine besondere Ursache“. - Unsere Beobachtung erstreckt sich natürlich nur auf die von PI. unmittelbar herangezogenen Quellen. 
bewußst gewordenen Gewissenspflicht der Quellennennung dadurch genügt zu haben glaubte, daß er den von ihm benutzten Autoren einen besonderen Ehrenplatz vor den einzelnen Büchern anwies ${ }^{21}$ ). Waren demnach die Indices dazu bestimmt, die ständigen Quellennachweise im Text zu ersetzen ${ }^{22}$ ), und werden hier die Quellen nur aus besonderen Gründen genannt, so dürfen wir nicht mit Brunn annehmen, daß die Plinianischen Autoren in der R e g e l erst bei ihrem ersten namentlichen Citat herangezogen worden $\operatorname{sind}^{23}$ ). In $\mathrm{der} \mathrm{Reg}$ el wird ein Autor im Text erst genannt worden sein, nachdem er in den vorhergehenden Partien des Buches bereits stillschweigend benutzt worden ist.

Die irrtümliche Einschätzung der namentlichen Citate liegt der ganzen Beweisführung Brunns zu Grunde, worin er der Hauptsache nach zu zeigen sich bemüht, Plinius habe in der ursprünglichen Redaction der Nat. hist. die Registerautoren den namentlichen Citaten entsprechend geordnet. Ich behaupte, daßs wir, gerade die Gültigkeit des Brunnschen Gesetzes vorausgesetzt, erhebliche Abweichungen in der Anordnung der Registerautoren von der der Textesautoren erwarten sollten. So wird, um nur ein Beispiel anzuführen, Verrius Flaccus im Index des siebenten Buches an erster, im Text ziemlich an letzter Stelle ( $\$ 180)$ genannt worden sein, weil er in den ersten Paragraphen stillschweigend benutzt worden ist ${ }^{24}$ ). In dieser Weise mögen noch manche Abweichungen

21) Neuerdings ist die Ansicht ausgesprochen worden, daß die In. dices ,einen untrennbaren Teil des ersten Buches bilden, dessen Wiederholung nur erfolge, um den Lesern das Nachschlagen bequemer zu machen" (Detlefsen Untersuch. S. 19). Indessen auch wenn nicht ausdrücklich bezeugt wäre, daß $\mathrm{Pl}$. die Listen in erster Linie den einzelnen Büchern beigegeben hat (n. h. XVIII 212 auctores prodidere ea quos praetexuimus volumini huic), so dürfte schon durch das Verbum praetexere (praef. 21) statt des gewöhnlichen praemittere ihr Charakter als den einzelnen Volumina zur Aufschrift bestimmte Ehrenregister genügend bezeichnet sein.

$\left.{ }^{2}\right)$ Vgl. Peter a. a. O.: „Im Text selbst das Eigentum eines jeden kenntlich zu machen, erschien $\mathrm{Pl}$. danach überflüssig".

$\left.{ }^{43}\right) \mathrm{De}$ ind. 2: tenendum porro Plinium, ubi primum auctorem aliquem exscripsit, non semper id addito nomine fecisse, womit offenbar die anfänglich stillschweigende Benutzung einer Quelle als Ausnahmeerscheinung bezeichnet wird.

24) Hierfür werden in der demnächst erscheinenden Quellenuntersuchung des 7. Buches entscheidende Argumente beigebracht werden. 
in der Reihenfolge der Registerautoren von der der namentlichen Citate im Text, in denen Brunn Störungen seines Gesetzes erblickte, aus Plinius' Absicht zu erklären sein, die Autoren in der Reihenfolge ihrer Benutzung anzuordnen. Also dürfen wir das Brunnsche Gesetz dahin modificieren, daß der Verfasser der Nat. hist. seine Quellenschriftsteller, unbekümmert um die namentlichen Citate im Text, in der Regel in derselben Reihenfolge in die Register eingetragen hat, wie sie ihm bei der Ausarbeitung der einzelnen Bücher Material geboten hatten? Allem Anschein nach hat sich unser Autor vielfach von diesem Grundsatz leiten lassen, leider aber in der Regel sich die Arbeit recht leicht gemacht, indem er seine Quellen und nicht nur die unmittelbar benutzten, sondern auch die 'aus Sammelwerken, Uebersetzungen und Citaten anderer ihm bekannt gewordenen' (Teuffel-Schwabe II 761 $\$ 313)^{25}$ ) mechanisch in derselben Reihenfolge in die Register eintrug, wie er sie im Text citiert fand ${ }^{26}$ ). Aus dieser Eigentümlichkeit der Indices aber, die Brunn recht eigentlich zu seiner Hypothese veranlaßit hat, folgt die Unbrauchbarkeit derselben für die Quellenforschung. Denn dann ist es nur unter die Ausnahmefälle zu rechnen, wenn Verrius Flaccus am Anfang statt der Stelle des namentlichen Citates im Text entsprechend am Ende des Registers des 7. Buches verzeichnet

Vorläufig sei es gestattet auf m. Diss. S. 16 A 11 zu verweisen. Brunn findet die römischen Quellen von B. VII, dem er jede Disposition abspricht, nullo certe ordine im Index aufgeführt (de ind. 11), und Mün. zer möchte in jener Divergenz des Verriuscitates "Spuren einer älteren Disposition des Buches" erkennen (Beiträge 40\%). "Diese Hypothese aber dürfte schon deshalb zu verwerfen sein, weil Verrius im T'ext für eine Sammlung plötzlicher Todesfälle citiert wird, eine die Merkwürdigkeiten der menschlichen Natur von der Geburt bis zum Tode umfassende Darstellung des Menschen aber schwerlich jemals mit einer Beschreibung des Todes begonnen haben wird.

25) Vgl. Brunn de ind. $46 \mathrm{ff}$.

26) Daher die häufig übereinstimmende Bezeichnung der Autoren im Text wie in den zugehörigen Indices: z. B. wird der in der Nat. hist. sonst als Timaeus, Timaeus Siculus oder Timaeus historicus erwähnte bekannte Geschichtsschreiber in den Indices der Bücher V und XVI den Citaten V 55 und XVI 82 entsprechend als Timaeus mathematicus, ähnlich Caelius Antipater in den Indices von Buch III und XXXI entsprechend den Citaten III 132 und XXXI 21 als Caelius, dagegen mit Namen und Beinamen im Index des II. Buches entsprechend dem Citat II 169 aufgeführt. 
ist. Dann muß jede Quellenuntersuchung damit rechnen, daß ein am Ende eines Index genannter Autor schon zu Anfang des betreffenden Buches stillschweigend verwendet worden ist, wie in der Tat schon Münzer den auf Grund des Citates IX 65 in der Mitte des Index genannten Fenestella bereits $\$ 11$, den gegen Ende des Index des XII. Buches genannten Claudius Caesar $\$ 10$ und 12, den gegen Ende des Index des XIV. Buches genannten Columella $\$ 52$, den in der Mitte genannten Varro $\$ 9$ und im XV. Buche gar $\$ 2$ benutzt sein läßt ${ }^{27}$ ). Es müssen also sämtliche in dem Register eines Buches genannten Autoren ohne Rücksicht auf das Brunnsche Gesetz als mögliche Quellen in Betracht gezogen werden. Allerdings finden sich in der Anordnung der Registerautoren noch genug Abweichungen von der der Textesautoren. Dieselben aber sind zweifellos zu einem nicht unerheblichen Teil der flüchtigen Arbeitsweise unseres Autors zuzuschreiben, der, wie wir sahen, selbst in einem einzelnen Paragraphen des Textes genannte Autoren in den Indices in anderer Reihenfolge verzeichnet, in den seltensten Fällen wohl der ursprünglichen Absicht des Verfassers, die Autoren in der Reihenfolge ihrer Benutzung anzuordnen.

Nach diesen Feststellungen darf das Brunnsche Gesetz, das nachgerade zu einer unerträglichen Fessel für die Quellenforschung geworden ist, wohl zum alten Eisen getan werden.

2.

Nun aber wären wir sehr im Irrtum, wenn wir glaubten, uns in der Untersuchung der Quellen eines Buches auf die in dem zugehörigen Index genannten Autoren beschränken zu dürfen. Bei der mechanischen Konstruktion der Indices, daf sich Plinius in der Regel von den Citaten hat leiten lassen, muß man fürchten, daß im Text eines Buches verwertete, aber nicht citierte Autoren später bei der Redaction der Indices unberücksichtigt geblieben sind. Schon Münzer hat die Beobachtung gemacht, daG im Text namentlich citierte Quellenschriftsteller und darunter eine unverhältnismäßig große Zahl

${ }^{27)}$ Beiträge S. 384. 391. 35 f. 270.

Philologug LXV (N. F. XIX), 4. 
unmittelbar benutzter ${ }^{28}$ ) in den zugehörigen Indices vermifit werden. Wie oft mögen erst Autoren übergangen sein, die im Text nicht citiert werden, aber stillschweigend benutzt worden sind! Das mußs, denke ich, den Quellenforscher vor jedwedem Zutrauen zu den einzelnen Indices warnen. Wenn z. B. Verrius Flaccus in der Nat. hist. nur siebenmal namentlich citiert, aber für drei von diesen sieben Citaten (IX 77, XXXIII 63. 111) in den zugehörigen Listen vermibt wird, so muf eine besonnene Quellenforschung damit rechnen, dafs sein Name in jedem einzelnen Register übergangen sein könnte, wie in Wirklichkeit bereits Münzer kein Bedenken getragen hat, Nachrichten des X. und XXXVI. Buches für Verrius Flaccus in Anspruch zu nehmen, obgleich der Name dieses Autors weder im Text noch in den Indices der genannten Bücher begegnet ${ }^{29}$ ). Wollen wir demnach für irgend eine nicht besonders beglaubigte Notiz der Nat. hist. die Quelle des Plinius ermitteln, so sind nicht nur die in dem zugehörigen Index, sondern sämtliche in den übrigen Indices verzeichneten Schriftsteller als mögliche Quellen in Betracht zu ziehen.

Die Untersuchung aber wird dadurch noch bedeutend erschwert, daß die Indices neben unmittelbaren auch sekundäre und sogar solche Autoren aufweisen, die in den betreffenden Büchern überhaupt nicht benutzt worden sind ${ }^{30}$ ). Man hat darin 'nicht eine Unehrlichkeit, sondern weitere Anwendung des vom Verfasser in dem Dedicationsschreiben ausgesprochenen Princips der Quellennennung auf die indirekten und vergleichsweise herangezogenen Quellen' sehen wollen ${ }^{31}$ ). Mir scheint, wer die oben ausgehobene Stelle des Programms unbefangen interpretiert, kann Plinius den Vorwurf nicht ersparen, dak ihn nicht so sehr, wie er uns glauben machen

28) Verrius IX 77, XXXIII 63. 111. Mucian XIV 54, XXI 33, XXVIII 29, XXXII 62 f., XXXIV 36 (Beiträge S. 129), wozu ich mir noch folgende Fälle notiert habe: Corbulo II 180, Aufidius VI 27, Claudius Caesar VII 35, Egnatius Calvinus X 34, Vitellius XI 187, Seneca XXIX 10.

$\left.{ }^{29}\right)$ Beiträge S. 305. 310 A 1.319.

30) Ueber die in den Indices der geographischen Bücher stets vereinigten Hygin, Vetus, Mela sind zu vergleichen Schweder Phil. LVI S. 284 und Münzer Beitr. S. 130 356, über Tarquitius und Aquila Münzer Beitr. S. 224. Vgl. auch m. Diss. S. 60 f. A 27.

31) Oehmichen Plin. Stud. S. 87. 
will, das Gefühl der Dankbarkeit gegenüber seinen Quellen, als die Begierde, mit seiner Belesenheit zu glänzen, zur Quellennennung in den Indices veranlafit hat.

Ueberhaupt ist der Gelehrteneitelkeit unseres Autors, die gelegentlich schon von Montigny bemerkt wurde ${ }^{32}$ ), in den bisherigen Beurteilungen seiner Arbeitsweise (vgl. Schanz II 379) zu wenig Beachtung geschenkt worden. Allerdings hat ihn Münzer mehr als einmal im Verdacht, daß er den Anschein erwecken wolle, als habe er selbständig aus den von ihm augenscheinlich nur aus zweiter Hand citierten alten Quellen geschöpft ${ }^{33}$ ). Dessenungeachtet aber trägt er kein Bedenken, Plinius als einen Geistesverwandten des großen Gelehrten und Antiquars M. Terentius Varro zu bezeichnen ${ }^{34}$ ). Im allgemeinen ist, soviel ich sehe, zuerst von Schweder, nach dessen Ansicht die geographischen Bücher der Nat. hist. einen Auszug aus einem verschollenen umfangreichen Werk darstellen, als er sich von Detlefsen auf Plinius' ausdrückliche Versicherung n. h. III 1 auctorem neminem unum sequar, sed ut quemque verissimum in quaque parte arbitrabor verwiesen sah, auf die Gelehrteneitelkeit unseres Autors aufmerksam gemacht worden, wie sie 'durch die sonderbaren Quellenregister und durch diejenigen Angaben in der Vorrede, in denen er sich seiner Studien und seiner groß̉en Belesenheit rühmt, für jeden Einsichtigen zur Genüge dargetan werde' ${ }^{35}$ ).

34) Quaestiones in C. Plin. Sec. nat. hist. de animalibus libros Bonn. 1844 S. 8.

$\left.{ }^{33}\right)$ Beitr. S. 27. 29. 191. 243. 279. 304. u. a.

${ }^{34)}$ Beitr. S. 423. Nach M. wäre freilich auch Varro nicht frei von Eitelkeit gewesen: „Die kleine Eitelkeit des greisen Memoirenschreibers, die sich in der Aufzählung seiner Ehrenzeichen verrät (n. h. XVI 7), befremdet im Altertum noch weniger als in manchen ähnlichen Werken neuer und neuster Zeit. Deshalb darf auch VII 115 unbedenklich aus derselben Quelle abgeleitet werden, wo neben der Verleihung der corona rostrata eine zweite dem Varro erwiesene außerordentliche Fhre erwähnt wird, daß nämlich seine Büste als die einzige eines Lebenden in der ersten offentlichen Bibliothek aufgestellt worden sei“ (Beitr. S. 277; vgl. S. 279). Dieser Auffassung gegenüber, die im Widerspruch steht mit allem, was wir von Varros Persönlichkeit wissen, werden die in der N. h. enthaltenen Ruhmesnotizen auf Varro sich leicht als Eigentum des Verrius Flaccus erweisen lassen.

${ }^{35}$ ) Phil. LIV S. 535. Vgl. S. 538: , wenn Pl. VI 149 angiebt, er folge hier hauptsächlich dem Demodamas, so hat er selbstrerständlich eine Schrift des Letateren nie vor Augen gehabt; er citiert ihn nach seiner römischen Quelle, und aus dieser stammt alles, was er über 
Ich möchte hier einige für Plinius' Gelehrteneitelkeit charakteristische Fälle anführen, die vielleicht geeignet sind, den im Rufe eines gelehrten Sammlers stehenden Verfasser der Nat. hist. zum reinsten Kompilator zu stempeln.

Obgleich unser Autor die in Form von Citaten griechischer Paradoxographen gegebenen Beispiele merkwürdiger Körpergestalt $n$. h. VII 9-32 nachweislich einer römischen Mittelquelle entnommen hat ${ }^{36}$ ), beruft er sich doch $\$ 8$ ganz naiv auf seine griechischen Gewährsmänner, als hätte er sie selbständig eingesehen: nec tamen ego in plerisque eorum obstringam fidem meam potiusque ad auctores relegabo qui dubiis reddentur omnibus, modo ne sit fastidio Graecos sequi tanto maiore eorum diligentia vel cura vetustiore.

Aehnlich erklärt er im folgenden Buche $\$ 43 \mathrm{f}$., sich in seiner Zoologie wesentlich an die von ihm selbst in e in en Auszug gebrachten 50 Bücher der AristoteJischen Tiergeschichte anschliefen zu wollen: quae $a$ me collecta in artum cum is quae ignoraverat quaeso ut legentes boni consulant, in universis rerum naturae operibus medioque clarissimi regum omnium (Alexanders des Großen) desiderio cura nostra breviter peregrinantes. Die Konkordanzen der Plinianischen Excerpte aus Aristoteles' Tiergeschichte nebst dem angeblich von unserem Autor selbst Hinzugefügten mit Plutarchs de sollertia animalium und Aelians de natura animalium lassen aber keinen Zweifel, daßs Plinius die Kenntnis des Aristoteles in der Hauptsache durch einen oder mehrere Sammelschriftsteller vermittelt worden ist ${ }^{37}$ ).

Demodamas dort angiebt. Wir aber können aus diesem Beispiel abnehmen, wie die Versicherung des Plinius III 1 zu verstehen ist, und welchen Wert sie hat".

${ }^{38}$ ) Nach der gewöhnlichen Ansicht Varro (Münzer Beitr. 17b, Rusch Stettin. Gymn. Progr. 1900 S. 7, Hosius Ausg. d. Gellius noct. Att. praef. LII adn. 2), wie ich glaube Quellenstudien III nachweisen zu können, Verrius Flaccus. Ebendaselbst wird gezeigt werden, daß auch Gellius Noct. Att. IX 4 nicht, wie man nach Mercklins Vorgange anzunehmen pflegt (Hosius a. a. O. praef. XXXIX. Schanz III 160, Peter d. geschichtl. Litt. üb. d. röm. Kaiserzeit I 126), aus Plinius, sondern PI.' Quelle geschöpft hat (Nettleship Lectures and Essays S. 256). Vorläufig sei auf meine Diss. S. 16 A. verwiesen.

37) Nach Münzer Beitr. $37 \mathrm{f.} 411$ durch Juba und Trogus (vgl. Birt de Hal. S. $135 \mathrm{ff}$.), nach Rusch a. a. $O$. durch Varro, nach meiner Ansicht durch Verrius Flaccus. Die gemeinsame Quelle von Ael. VII 
Auf einen besonders starken Fall endlich habe ich schon in meiner Dissertation hingewiesen: XXXIII 14 Romae ne fuit quidem aurum nisi admodum exiguum longo tempore, certe cum a Gallis capta urbe pax emeretur, non plus quam mille pondo effici potuere, nec ignoro duo milia pondo auri perisse Pompeio III cos. e Capitolini Iovis solio a Camillo ibi condita, et ideo a plerisque (Varro und den ihm Folgenden wie Dionys. Vgl. Mommsen Röm. Forsch. II 330 A. 75) existimari duo milia pondo conlata. sed quod accessit ex Gallorum praeda fuit detractumque ab iis in parte captae urbis delubris (15) ... apparet ergo Gallorum templorumque tantundem nec amplius fuisse. quod quidem in augurio intellectum est, cum Capitolinus duplum reddidisset ... (16) ergo ut maxime duo milia tantum pondo, cum capta est Roma, anno CCCLXIV fuere ... in eadem post annos CCCVII, quod ex Capitolinae aedis incendio ceterisque omnibus delubris C. Marius filius Praeneste detulerat. - Ich habe gezeigt, daf das Jahr der Einnahme Roms nicht, wie es scheint, nach varronischer, sondern capitolinischer Aera datiert ist, dafis mithin die mit der Maske eines gewiegten Kritikers vorgetragene Polemik gegen Varro nicht das eigene kritische Urteil unseres Autors

44 und Plut. XVII 3 muß nämlich bereits die Schriften des Königs Juba benutzt haben (Wellmann Herm. XXVII 398). Nun verdanken Plin, Ael. u. Plut. eine Reihe von Aristotelescitaten zweifellos einer gemeinsamen Mittelquelle (Wellmann a. a. O. S. $389 \mathrm{ff} .$, Rusch a. a. O.). Also scheinen alle drei aus einer nach Juba schreibenden Quelle geschöpft zu haben. Rusch hat die Resultate seiner Arbeit dadurch zersplittert, daß er den nach Juba anzusetzenden Autor als gemeinsame Quelle Aelians und Plutarchs gelter lassen, hingegen für Plinius an der unmittelbaren Benutzung der Schriften Jubas festhalten möchte: die gemeinsame Quelle aller drei Schriftsteller sei Varro, die gemeinsame Quelle Aelians und Plutarchs Alexander Myndios gewesen. Nun stützt sich R.' Varrohypothese in der Hauptsache darauf, daß er nach Münzers Vorgang die Beispiele merkwürdiger Körperbeschaffenheit VII $9 \mathrm{ff}$. auf Varro zurückführt. Läßt sich aber hier Verrius als Quelle erweisen (vgl. Anm. 36), so könnte R.' komplicierte Hypothese leicht durch eine einfachere ersetzt werden, da Verrius die Gelehrsamkeit der Varro und Juba in einer Person repräsentiert. Vgl. das Verriuscitat VIII 17 (hierzu m. Diss. S. 59 f.), Charisius gramm. lat. I p. 102,10 und die litterargeschichtliche Bemerkung über eine Schrift des Königs Juba VI 141 (hierzu Anm. 40). - Indessen ist eine Entscheidung über die Persönlichkeit des von Pl. benutzten Sammelschriftstellers, die erst durch eine Behandlung des Quellenproblems Mela-Plinius.Solin gewonnen werden kann, für dje oben in Betracht kommende Erkenntnis der Arbeitsweise unserers Autors nicht von Belang. 
enthält, wie er uns glauben machen will (nec ignoro), sondern der ganze Abschnitt einem Schriftsteller der ersten Kaiserzeit (Verrius Flaccus) verdankt wird ${ }^{38}$ ).

Es dürfte überflüssig sein, weitere Beispiele für die Gelehrteneitelkeit unseres Autors zu sammeln. Schon die hier vorgelegten, die sich in nichts von regulären Plagiaten unterscheiden, lehren zur Genüge, was wir von Plinius' programmatischer Versicherung, er habe die 20000 Merkwürdigkeiten der Nat. hist. aus 2000 den entlegensten Gebieten der Weltlitteratur angehörigen Rollen ex exquisitis auctoribus centum zusammengelesen (praef. 17), und derartigen Erklärungen über selbständige Benutzung alter römischer und griechischer Quellen an zahlreichen Stellen des Textes zu halten haben ${ }^{39}$ ). Diese ständigen Versicherungen, soweit sie Plinius nicht aus seiner Vorlage herübergenommen hat ${ }^{40}$ ), sind

88) Diss. S. $23 \mathrm{ff}$. Zu den hier beigebrachten Argumenten ist noch zu vergleichen Mommsen Gesch. d. röm. Münzwes. S. 401 A. 106 (das Geschichtchen von dem Diebstahl unter Pompeius' alleinigem Konsulat nsicher eine Parteianekdote der Caesarianer ") u. Röm. Forsch. II 238, ferner die ausführliche gelehrte Polemik gegen Varro n. h. XIII $84 \mathrm{ff}$. Dal3 Pl. diese einem Antiquar der tiberischen Zeit verdankt, lehrt ein Vergleich des Plinianischen Berichts über die Papyrusstaude und ihre Fabrikation XIII 74-80 mit dem entsprechenden in Isidors Orig. VI 10. Pl.' Bemerkungen über das von Isidor, der nur 7 Papiersorten anführt, nicht erwähnte Claudiuspapier $\$ 79 / 80$ nämlich kennzeichnen sich schon äußerlich deutlich als eine die Darstellung ganz ungehörig unterbrechende Einlage, indem an den Schlußsatz von $\$ 78$ 'praeterea spectantur in chartis tenuitas, densitas, candor, levor' sich $\$ 81$ 'scabritia levigatur dente conchave' unmittelbar anschließt und Pl. sogar vergessen hat das 'XIII digitorum optimis' seiner Vorlage $\$ 78$ mit Rücksicht auf $\$ 80^{\circ}$ 'praelata omnibus Claudia' in 'XIII digitorum Augustaeae et Livianae' abzuändern.

39) Noch in einer seiner jüngsten Schriften hat sich Detlefsen durch Plinius' Aussagen über seine Quellen mystificiren lassen, wenn er die einschlägigen Untersuchungen Schweders, ohne sie zu nennen, von vornherein mit der Begründung ablehnt: „Auf keinen Fall scheint Pl. ein bloßer abbreviator eines einzelnen, ausführlichen Gewährsmannes zu sein, sondern regelmäßig mehrere Schriften zur Herstellung seines Textes benutzt zu haben" (Die Beschreibung Italiens in der Nat. hist. des Pl. Leipzig 1901. Sieglins Forsch. z. alt. Gesch. u. Geogr. Heft 1,1). Vgl. die Recension von Reitzenstein, Deutsch. Litt. Zeit XXV (1904) S. 2365: „Die Hauptquelle des Mela, auf welche Schweder immer wieder hingewiesen hat, ist nicht berücksichtigt. Für Mela dürfen wir eine einheitliche Quelle aus dem Beginn unserer Zeitrechnung annehmen".

40) Der VII 8 ausgesprochene Grundsatz der Quellenbenutzung kehrt ganz ähnlich XXV IIl 2 wieder. Da sich die einleitenden Kapitel beider Bücher auf Verrius zurückführen lassen (vgl. Anm. 36 u. m. 
lediglich rhetorisch aufzufassen. Denn es gehörte zum Handwerk der rhetorischen Schriftsteller - und wir wissen, daß Plinius im Zeitalter der Rhetorik gelebt, selbst drei Bücher über das Studium der Beredsamkeit geschrieben hat und daß er von Quintilian (inst. III 1, 21), seinem jüngeren Zeitgenossen, zu den rhetorischen Schriftstellern gerechnet wird - ihren Werken den Anstrich möglichst gediegener Gelehrsamkeit zu verleihen, weshalb wir auch weniger Plinius selbst für sein Verhalten verantwortlich machen dürfen, als den Geist der Rhetorik, der den ursprünglichen Wahrheitssinn der Römer vergiftet hat.

Hiernach kann es keinem Zweifel mehr unterliegen, daß3 wir in den sonderbaren Quellenverzeichnissen wesentlich rhetorische Machwerke zu erblicken haben. Auch die oft bemerkte Differenz zwischen der Angabe unseres Autors in der Vorrede, er habe ex exquisitis auctoribus centum geschöpft (praef. 17) und der fast fünfmal so grossen Zahl von Autoren in den Indices dürfte jetzt nicht mehr problematisch sein. Man hat unter den exquisiti auctores die direkten, unter den übrigen die indirekten und vergleichsweise herangezogenen Quellen verstehen wollen ${ }^{41}$ ). Ohne Zweifel aber will Plinius die in den Listen genannten Autoren ausnahmslos wirklich eingesehen und in der Naturgeschichte verwertet haben. Jener Widerspruch wird also daraus zu erklären sein, daß unser Autor sich weder selbst die Mühe gegeben hat, die Autoren in den Indices nachzuzählen - wir wissen, dab Plinius sie nicht in ihrer Gesamtheit veröffentlicht, sondern den einzelnen Büchern als Aufschrift beigegeben hatte, und haben uns überdies von der flüchtigen Herstellung der Register über-

Diss. S. 47 A. 10), dürften jene quellenkritischen Bemerkungen, zumal sie sich auch bei Solin finden (vgl. Peter die geschichtl. Litt. I 134), für den sich gleichfalls Verrius' Encyclopädie als Hauptquelle wird nachweisen lassen, bereits in der Quelle gestanden haben. Auch VI 141, wo Pl. in gedankenlosem Anschluß an einen Autor der tiberischen Zeit den Isidor von Charax (statt Mela) als jüngsten geographischen Schriftsteller bezeichnet (vgl. m. Diss. S. 51), müssen die unmittelbar folgenden quellenkritischen Bemerkungen aus derselben Quelle stammen. - Vgl. Schweder Phiı. LIV S. 538 aber die indirekte Benutzung des Demodumas.

41) Brunn de ind. 46, Münzer deutsche Litt. Zeit. 1900 S. 997, Schanz II 2, 378 . 
zeugt - noch einem seiner Leser diese Arbeit zugetraut hat, daß er mithin der Meinung hat sein können, nicht viel mehr oder weniger als etwa 100 Autoren in den Registern aufgeführt zu haben, oder aber, was weniger wahrscheinlich ist, daß er in der Zuschrift an Titus absichtlich nur eine beschränkte Zahl von Autoren als seine Quellen bezeichnet, weil er fürchten mukte, dafi ihm die 500 Autoren niemand so leicht glauben würde ${ }^{42}$ ).

Aber wir dürfen weitergehen und behaupten, dak Plinius die Indices nicht allein aufgestellt hat, um mit dem Schein großer Gelehrsamkeit zu prunken, sondern auch in der Absicht, eine Kontrolle, die zur Erkenntnis der von ihm benutzten Hauptquellen führen könnte, möglichst zu erschweren.

Es ist eine bisher noch nicht genügend beachtete Erscheinung, daß sich in der Nat. hist. für die zahlreichen Nachrichten aus der ersten Kaiserzeit keine direkten Quellencitate finden, die älteren Quellenschriftsteller hingegen überaus häufig, u. a. Nepos über zwanzig, Varro über siebenzig, Cato ïber neunzig Mal, im Text citiert werden. Münzer geht über dieses Problem mit den Worten hinweg, daß Plinius „nicht sagen wollte, welche historischen Darstellungen er für das Jahrhundert seit Caesars Tode benutzte " (Beiträge S. 250). "Hier hat Plinius absichtlich und bewußt", sagt er an einer anderen Stelle (Beiträge S. 131), „ eine ganze Kategorie seiner Quellen nicht genannt ${ }^{*}$. Die oben gewonnene Einsicht in den Charakter der Schriftstellerei unseres Autors giebt uns aber, denke ich, den Schlüssel auch zur Lösung dieses Problems.

42) Mit Unrecht ist auf die Bezeichnung der auctores centum als exquisiti ein besonderes Gewicht gelegt worden, als habe sie Pl. dadurch ausdrücklich als eine Elite seiner Quellen bezeichnet. Gegen diese Auffassung spricht neben dem rhetorischen Character der ganzen $\nabla_{\text {or- }}$ rede, in der sich Pl. alle Mühe gibt, mit seiner Belesenheit zu glänzen, insbesondere der Umstand, dal an der betreffenden Stelle gerade den Zahlworten ein besonderer Nachdruck verliehen wird: praef. $17 X X$ milia rerum dignarum cura lectione voluminum circiter II milium quorum pauca admodum studiosi attingunt propter secretum materiae, ex exquisitis auctoribus centum inclusimus $X X X V I$ voluminibus, adiectis rebus plurimis . nec dubitamus multa esse quae et nos praeterierint. Das Adj. exquisiti darf also nur als die Vorzüglichkeit der benutzten stattlichen Reibe von Autoren im allgemeinen bezeichnendes Epitheton ornans gefaßt werden. 
Nachdem wir nämlich den Verfasser der Nat. hist. von der Höhenstellung eines gelehrten Sammlers in die Klasse der gewöhnlichen Kompilatoren eingereiht haben, liegt es nahe, seine Citiermethode mit derjenigen des geistesverwandten Verfassers der Noctes Atticae zu vergleichen. Dieser pflegt bekanntlich seine Hauptgewährsmänner zu verschweigen, seine indirekten Quellen hingegen gerade so zu citieren, wie er sie bei jenen vorfand (Mercklin, d. Citiermethode u. Quellenbenutzg. d. Aul. Gellius 1860). Das oben bezeichnete Problem findet also seine einfache und, wie ich glaube, einzig mögliche Erklärung darin, daßs die Hauptquellen des Plinius für die Nachrichten aus der ersten Kaiserzeit zugleich seine Hauptquellen waren, die er für ihre eigene Zeit stillschweigend ausschrieb, während er die darin angeführten Quellen citiert, als habe er sie selbst eingesehen. Hier begegnen wir uns mit dem Ergebnis des ersten Teils der Quellenstudien, daf der Hauptrom der unmittelbaren Quellender Nat. hist. nicht, wie es die übliche Anschaungist, der Zeit der römischen Republik, sondern der ersten Kaiserzeit angehört.

Also Schweder hat Recht, wenn er annimmt, daß Plinius "für viele, vielleicht für die meisten in der Nat. hist. behandelten Disciplinen umfassende Hand- und Lehrbücher, die für uns verloren und verschollen sind, vorfand, auszog und diese Auszüge mit verhältnismässig wenigen Zusätzen, den Früchten eigenen Studiums oder besser eigener Lektüre, bereichert in einem Gesamtwerk zu einer Encyclopädie vereinigte" (Phil. LIV S. 538). Aehnlich hat sich in der Einleitung seines Werkes Münzer ausgesprochen: „Einen Ersatz für die Schwierigkeit des Nachprüfens von Citaten gaben die antiken gelehrten Arbeiten, indem sie ihr Material vielfach vollständig vor dem Leser ausbreiteten und alle Belegstellen ausschrieben ${ }^{43}$ ). $\mathrm{Zu}$ allen Zeiten sind solche Werke weniger verbreitet und infolgedessen leichter dem Untergang ausgesetzt gewesen, als die von ihnen abhängigen mehr populär gehaltenen. Eines

43) Freilich hat M., um die Schriftstellerehre des "gelehrten Sammlers " zu retten, in seinen Einzeluntersuchungen sehr oft „Nachprüfung älterer Quellencitate seitens unseres Autors angenommen. 
der letzteren haben wir in der Naturgeschichte des Plinius vor uns, in die sich manche der ersteren ergossen. S e in e Hauptquellen waren zusmmenfassende gelehrte Arbeiten, die nicht allzu lange vor seiner eigenen $Z$ eit den Bestand der Wissenschaften fixiert hatten " (Beiträge S. 8).

Nun ist, namentlich von Detlefsen, immer wieder auf das Zeugnis Plinius' des Jüngeren über die ausgedehnten Studien seines Oheims verwiesen und die Ansicht ausgesprochen worden, daß die darin als Frucht seines Sammeleifers erwähnten 160 auf beiden Seiten eng beschriebenen Buchrollen als Grundlage der Nat. hist. zu betrachten seien ${ }^{44}$ ). Das Zeugnis des Neffen aber spricht keineswegs zu Gunsten unseres Autors. Dieser pflegte, heißst es in jenem Brief, alles zu lesen und zu excerpieren, was ihm in die Hände kam: dicere etiam solebat nullum esse librum tam malum, ut non aliqua parte prodesset. Und mit welcher Hast hat er gelesen oder sich vorlesen lassen! Den grössten Teil seiner Zeit mulite er den Staatsgeschäften widmen: ante lucem ibat ad Vespasianum .. inde ad delegatum sibi officium (vgl. n. h. praef. 18). Nach der Mahlzeit erst iacebat in sole, liber legebatur, annotabat excerpebatque. Auch las er wohl in einer Sänfte, in der er sich durch die geräuschvollen Straßen der Hauptstadt tragen ließ, oder auf Reisen, wo ein Schreiber mit einem Buche und einer Tafel an seiner Seite saß. Kann ein solcher Mann, fragt man mit Recht ${ }^{45}$ ), im stande gewesen sein, bei dem Gelesenen denkend $\mathrm{zu}$ verweilen, um es geistig zu verarbeiten? Wenn der jüngere Plinius, der in Wirklichkeit für die Sammeltätigkeit des Oheims nicht viel übrig gehabt zu haben scheint ${ }^{46}$ ), von dem Ergebnis der Studien unseres Autors berichtet: hac intentione tot ista volumina peregit electorumque commentarios

44) Plin ep. III 5. Vgl. Detlefsen Untersuchungen S. 16f., Schanz II 2, 374, Peter d. geschichtl. Litt. I 121.

43) Schweder Phil. LIV S. 533.

46) Ueber seine geringe Vertrautheit mit dem Inhalt der Naturalis historia vgl. Schanz II 2, 374 A. 5. Bezeichnend ist auch die Anekdote aus der Studentenzeit des Epistolographen g. E. des Briefes: repeto me correptum ab eo cur ambularem: 'poteras, inquit, has horas non perdere', nam perire omne tempus arbitrabatur, quod studiis non impenderetur. 
centum sexaginta mihi reliquit, so mögen diese Rollen einen schönen Wust von Excerpten enthalten haben. Doch wird kein Einsichtiger glauben, daß aus solchem Sammelsurium jemals "ein Studierlampenbuch wie die Naturgeschichte" (Mommsen, Herm. XIX 648) hat hervorgehen können.

Also auch das Zeugnis Plinius' des Jüngeren zeigt uns, wie armselig es mit den eigenen Studien unseres Autors bestellt war. Mithin ist Schweders Kombination unabweislich, daß umfangreiche gelehrte Arbeiten, die Plinius auszog, den Grundstock der Nat. hist. bilden und daß den eigenen Excerptenrollen des Verfassers nur wenige Notizen angehören, mit denen er jene Auszüge (vielfach an wenig passender Stelle) bereicherte ${ }^{47}$ ). Es liegt daher nahe, die Entstehungsgeschichte der Nat. hist. mit derjenigen der Periegese des Pausanias zu vergleichen, der sich gleichfalls „weniger an seine Notizen und dasjenige, was er mit eigenen Augen beobachtet hatte, gehalten hat als an den reichlicheren Stoff, den ihm die damals landlïufigen, encyclopädischen Handbücher boten “ (Christ, Gesch. d. griech. Litt. ${ }^{4}$ S. 722).

Wollen wir jetzt die Verfasser der von Plinius ausgezogenen gelehrten Arbeiten ermitteln, so steht nach dem Vorhergehenden so viel fest, dak sie mit denjenigen Quellen identisch sind, denen die Nachrichten aus der ersten Kaiserzeit verdankt werden. Da sich aber für diese Nachrichten im Text keine direkten Quellencitate finden, so erhebt sich die Frage, ob die betreffenden Autoren vielleicht in den Indices verzeichnet sind. Münzers Ansicht geht dahin, Plinius habe "anscheinend absichtlich die Namen dieser Quellen nicht in seine Register aufgenommen " ${ }^{48}$ ). Nachdem wir aber die in

47) Vgl, zu Schweder a. a. O. S. 538 Münzer Beitr. S. 10: „In solchen Fällen (wo Pl. ,aus neueren Werken nur ganz vereinzelte Notizen, ja zuweilen aus einem Autor nur eine einzige * bringt) muß man sich der Schilderung erinnern, die der jüngere $P l$. von der unermüdlichen Lesewut seines Oheims entwirft. Die Excerpte und Notizen, die dieser sich machte oder machen ließ, während ihm ein Buch vorgelesen wurde, waren zum großen Teil keine fortlaufenden Auszüge und Referate sondern Lesefrüchte . . sie stehen in der $N$. H. in vielen Fällen am Anfang oder am Ende von Abschnitten, die sonst wesentlich eine in sich geschlossene Einheit darstellen, sind auch mitunter recht un. geschickt eingeschoben".

${ }^{48}$ ) Beiträge S. $385 \mathrm{Vgl}$. S. 131: „Vollständig versagen die Indices für die Historiographie der Kaiserzeit". 
tiberischer Zeit entstandenen Rerum memoria dignarum libri des Verrius Flaccus als eine Hauptquelle der Nat. hist. kennen gelernt haben ${ }^{49}$ ), also nicht daran zu zweifeln ist, daß ein großer Teil der Nachrichten aus der ersten Kaiserzeit diesem Quellenwerk verdankt wird, kann Plinius wenigstens für 10 von den 36 Indices, in denen der Name des Verrius erscheint $(3,7,8,14,15,18,28,29,34,35)$, von dem Verdacht des absichtlichen Verschweigens seiner Quellen und zwar seiner Hauptquellen, wie wir gesehen haben, entlastet werden. Auch wird das Versagen der Indices für die Nachrichten aus der ersten Kaiserzeit hin und wieder der Oberflächlichkeit unseres Autors bei der Herstellung der Listen zur Last gelegt werden dürfen, da er seine Quellen in derselben Reihenfolge, wie er sie im Text fand, in die Listen einzutragen pflegte, woraus wir geschlossen haben, dak Autoren, die, wie anscheinend diejenigen für die Nachrichten aus der ersten Kaiserzeit, im Text nur stillschweigend benutzt worden waren, in den Indices leicht unberücksichtigt geblieben sind. Läßst uns nun aber die Mehrzahl der Autorenregister bei der Ermittelung der Hauptquellen tatsächlich im Stich, insbesondere gleich die Listen der Bücher 2, 4, 5, 6, obwohl der Index des zweiten Buches noch für besonders zuverlässig gehalten wird ${ }^{50}$ ) und auch mit Recht gehalten werden sollte, so ist hierfür nicht mehr Flüchtigkeit, sondern sicherlich die Absicht des Verfassers verantwortlich zu machen, die Leser von der Erkenntnis seiner Hauptquellen abzulenken ${ }^{51}$ ). So bestätigt sich der Verdacht von Schweder, es habe Plinius nicht gefallen, seine Leser über die wirklich von ihm benutzten Quellen aufzuklären, er habe vielmehr seine Hauptquellen überall in Dunkel gehüllt ${ }^{52}$ ). Eigentümlich aber berühṛt es uns jetzt, wenn wir in der Vorrede unseren Autor es als die Pflicht eines anstän-

49) Vgl. m. Diss. S. $55 \mathrm{ff}$.

Бо) Münzer Beitr. S. 162.98.

61) Das zweite Buch enthält neben anderen Nachrichten aus augustisch-tiberischer Zeit (\$\$ 96. 168. 178. 200), deren Quellen bisher nicht ermittelt werden konnten, auch einige Notizen aus der Autobiographie des Augustus (\$S 24.93 f. 98), die Pl. zweifellos einer , absichtlich “ im Index nicht genannten Mittelquelle verdankt (Münzer Beitr. S. 250).

${ }^{52}$ ) Phil. LIV S. $532 \mathrm{f}$. Vgl. d. fingierten Quellenangaben in den Noctes Atticae. 
digen Mannes bezeichnen sehen, seine Quellen zu nennen: praef. 23 obnoxii profecto animi et infelicis ingenui est deprehendi in furto malle quam mutuum reddere cum praesertim sors fiat ex usura.

\section{4.}

Nachdem wir uns nunmehr von den Fesseln der gewöhnlichen Auffassung freigemacht haben, ist es ohne weiteres evident, daß̧ die Indices für den Quellenforscher nicht mehr den Ausgangspunkt geschweige die Basis der Untersuchung bilden dürfen. Wir sind in der Hauptsache auf Analyse und Vergleichen des Textes mit den uns erhaltenen encyclopädischen, fachwissenschaftlichen oder sonstigen sich mit dem Inhalt der Nat. hist. berührenden Schriften der römischen Kaiserzeit angewiesen.

"Die wichtigste Hülfe jeder Quellenuntersuchung ", betont A. v. Gutschmidt ${ }^{53}$ ) mit Recht, „wird immer die innere Kritik der Berichte gewähren“. Wesentlich auf einer solchen beruht die demnächst erscheinende Quellenuntersuchung des siebenten Buches der Nat. hist., das sich im Grossen und Ganzen als ein Auszug aus dem entsprechenden Teil der Verrianischen Encyclopädie herausstellen wird, während die sich hieran anschließende Behandlung des Quellenverhältnisses zwischen Mela und Plinius (n. h. III-VI), in deren Chorographien wir gleichfalls Auszüge aus dem Verrianischen Werk erkennen werden, beide Methoden (Analyse des Textes und Quellenvergleichung) verbindet, doch im Gegensatz zu Schweder, der die Concordanzen der beiden Autoren in den Vordergrund stellt, unter vorzüglicher Berücksichtigung des Pliniustextes, da dieser die Angaben der gemeinsamen Quellenschrift "nicht nur ausführlicher, sondern auch getreuer" (Schweder a. a. O. S. 551) wiedergibt. Nach einer Untersuchung des zweiten Buches, als dessen Hauptquelle wieder Verrius erwiesen wird, hoffe ich dann noch binnen kurzem eine Behandlung des Quellenproblems Mela-Plinius, Solin, Isidor vorlegen zu können, die nach-

53) Kl. Schriften I 14. 
weislich alle aus der Verrianischen Encyclopädie geschöpft haben ${ }^{54}$ ).

Bevor wir aber in die Untersuchung der einzelnen Bücher der Nat. hist. eintreten, möchte ich noch den Versuch machen, von allgemeinen Gesichtspunkten aus die Abfassungszeit der Hauptquellen der Plinianischen Encyclopädie etwas enger, als bisher geschehen ist, zu umgrenzen. Soviel könnte wohl nach den bisherigen Darlegungen schon als erwiesen gelten, daß3 der Hauptstrom der unmittelbaren Quellen nicht dem Zeitalter der letzten Republik, sondern der ersten

54) Daß Solin den Hauptbestand seiner geographisch geordneten Kuriositätensammlung nicht einem erweiterten und umgearbeiteten Plinius, dem unbekannten Verfasser der von Mommsen konstruierten chorographia Pliniana, sondern einer chorographia antepliniana, die auch Mela vorlag, verdankt, ist mit zum Teil guten Gründen schon vor $10 \mathrm{Jahren}$ von Columba angenommen worden (Rassegna di antichità class. vol. I 1896 fasc. $1-2$ : le fonti di Giulio Solino). Es werden von ihm Solinstellen beigebracht, die sich nur teilweise mit Pl. berübren, dagegen mit ähnlichen Notizen bei Strabo, Seneca in den Naturales quaest., Josephus, Tacitus in d. Germania, Aelian, Ammian, Augustin nicht nur im Wortlaut, sondern auch in sachlichen Angaben übereinstimmen, und daraus wird für $\mathrm{Pl}$., Solin und den jedesmal herangezogenen der oben genannten Schriftsteller eine gemeinsame Quelle postuliert. Es können aber auch noch Velleius, Dioscorides u. a. zum Vergleich berangezogen werden. Was Tacitus' Germania betrifft, deren Hauptquelle bisher nicht ermittelt werden konnte, so sind darin schon von Manitius (Forsch. z. deutsch. Gesch. XXII 1882 S. $417 \mathrm{ff}$.) zahlreiche Berührungen mit Melas Chorographie aufgezeigt worden. Während aber M. daraus auf Benutzung Melas in der Germania geschlossen hat, läßt sich für Tacitus, Mela. Plinius, Solin eine gemeinsame Quellenschrift nachweisen. Ueber $Z$ eit und Autor der von ihm als 'corografia varrosallustiana' (a. a. 0 . S. 116) bezeichneten gemeinsamen Quellenschrift hat Columba nichts Näheres ermitteln können. $\mathrm{Zu}$ Gunsten unserer Verriushypothese aber sei hier nur auf einige Argumente allgemeinerer Art hingewiesen, wodurch das Ergebnis der späteren Untersuchungen keineswegs praeiudiciert werden soll: daß schon der Titel von Solins Werk (Collectanea rerum memorabilium) an den Titel der Verrianischen Encyclopädie (Rerum memoria dignarum libri) erinnert und daß Solin ebenso wie Verrius (vgl. m. Diss. S. $59 \mathrm{ff}$.) nach capitolinischer Aera datiert, obgleich zu seiner Zeit allgemein varronisch gerechnet wurde, weshalb die Annahme Mommsens, Solin habe eine Tabelle des Bocchus benutzt, wenig Wahrscheinlichkeit für sich hat. - Was endlich die Concordanzen von Isidors Origines mit Pl. und Sol. betrifft, so ist auch Columba der Meinung, I. habe aus PI. und Sol. geschöpft, obgleich bereits Dressel in einer Col. anscheinend unbekannt gebliebenen Abhandlung über die Quellen Isidors (Rivista di filologia vol. III 1875 S. 232) bei Isidor aus Varro und Sallust stammendes Gut aufgezeigt hat, das sich weder bei Plin. noch Sol. findet, also auch aus der 'corografia varrosallustiana' stammen dürfte. Das alles wird noch eingehender nachgewiesen werden. 
Kaiserzeit angehört. Als terminus post quem wäre demnach ohne weiteres etwa das Todesjahr des M. Terentius Varro (27 v. Chr.) zu bezeichnen. Dennoch dürfte eine unsere Auffassung von dem Wert der Autorenlisten und namentlichen Citate bestätigende, allein auf der inneren Kritik der Berichte beruhende Untersuchung am Platze sein, die beweist, dafs der große Gelehrte der Republik tatsächlich keine Hauptquelle für Plinius gewesen ist.

Den meisten Gelehrten gilt noch heute Varro mit seiner erstaunlichen Gelehrsamkeit als Hauptquelle für die Mehrzahl der in der Nat. hist. behandelten Disciplinen, da sein Name in fast allen Autorenregistern und sehr häufig im Text genannt wird. Allein aus der Beurteilung gewisser Varro nahestehender Persönlichkeiten in der Nat. hist. dürfte ein untrüglicherer Schluf auf den Umfang der Benutzung Varronischer Schriften zu gewinnen sein. Ich behaupte nämlich: wäre Varro in dem Sinne, wie es die übliche Anschauung ist, Hauptquelle unseres Autors gewesen, so müßte es befremdlich erscheinen, daf Pompeius (ebenso wie sein Sohn Sextus) in der Nat. hist. so auffallend mifgünstig beurteilt wird, da doch Varro Jahrzehnte hindurch die Partei des grossen Pompeius "mit ausharrender Treue festgehalten, dessen Vertrauen genossen, dessen Pläne im einzelnen gekannt und tätig gefördert, dessen Erfolge und Unterliegen geteilt hatte " (Ritschl, Opuse. III 436). Allerdings kann man bei Münzer lesen: „Plinius, der so viel von Varro gelernt hat, zeigt auch eine Bewunderung und Vorliebe für Pompeius, die uns in Erstaunen setzt " (Beiträge S. 281). Aber gerade das Gegenteil ist richtig; überall, wo in der Nat. hist. Urteile über die Persönlichkeit des P. begegnen, fallen sie zu seinen Ungunsten aus. So wird VII 53 das berühmte os probum nicht ohne Ironie mit den Physiognomien einiger Plebejer verglichen: Magno Pompeio Vibius quidam e plebe et Publicius etiam servitute liberatus indiscreta prope specie fuere similes, illud os probum reddentes ipsumque honorem eximiae frontis ${ }^{55}$ ). Wie man hier von einer "günstigen Darstellung des P." sprechen kann

${ }^{55}$ ) Vgl. Solin I 81 p. 20, $10 \mathrm{M}^{2}$ : ut Romani Vibium Pompeii nomine, Pompeium Vibio vocabulo cognominarent. 
(Beitr. S. 111 ob.), ist mir unverständlich. Aehnlich findet Münzer in der Schilderung der Großstaten des Caesar und Pompeius VII 91-99, ,eigentlich nur den Ruhm des Letzteren verkündet" (Beitr. S. 281): „Nach der ausführlichen und breiten Schilderung seiner Großstaten fällt nur am Schluß (VII 99) ein mattes Lob für seinen Ueberwinder ab, wie auch kurz vorher sein leichter Sieg über die Piraten weit über Caesars sämtliche kriegerische Erfolge gestellt worden war (VII 93). Was überhaupt bei Caesar nach Verdienst gewürdigt wird, sind schlieklich nur vigor animi, clementia und magnitudo animi u. s. f." Auch hier hat M. die Tendenz der Schilderung nicht beachtet. Velleius, der selbst Militär gewesen ist, rühmt unter Caesars Verdiensten gleichfalls weniger seine kriegerischen Erfolge oder seine politischen Reformen als seine clementia und seine magnanimitas ${ }^{56}$ ). So vermeidet es Plinius' Quelle, wie aus $\$ 92$ praeter civiles victorias undecies centum et nonaginta duo milia hominum occisa proeliis ab eo non equidem in gloria posuerim, tantam etiamsi coactam humani generis iniuriam, quod ita esse confessus est ipse bellorum civilium stragem non prodendo hervorgeht, absichtlich, die Kriegstaten des Dictators einzeln aufzuzählen, weil in den Bürgerkriegen so viel Blut geflossen ist, und rechnet es ihm lieber zum Ruhme an, daß er die Zahl der in den Bürgerkriegen Gefallenen nicht öffentlich bekannt machte. Dennoch läßt sie uns nicht im Zweifel, wem von den beiden Rivalen sie den größeren Kriegsruhm zuerkennt. Wenn nämlich nach einer Aufzählung der Kriegstaten des Pompeius von Caesar gesagt wird: si quis e contrario simili modo velit percensere Caesaris res, qui maior illo apparuit, totum profecto terrarum orbem enumeret, quod infinitum esse conveniet (\$ 99), so darf man darin nicht mit Münzer ein mattes Lob des großen Dictators, sondern die entschiedenste Anerkennung seines einzig dastehenden, keines Kommentars bedürftigen Kriegsruhms erblicken ${ }^{57}$ ). Endlich ist aus der breiteren Schilderung, welche

$\left.{ }^{56}\right)$ Vell. II 52, 6. 56, $1 \mathrm{ff}$. Vgl. II 55, 2. 57, 1 und die entsprechende Schilderung der clementia Octavians II 86, 2. 87, 2. RGDA I $15 \mathrm{M}^{2}$. III $7 \mathrm{ff}$., IV $31 \mathrm{ff}$. $\mathrm{M}^{2}$.

$\left.{ }^{67}\right)$ Vgl. Vell. II 42, 1: longum est narrare, quid et quotiens ausus sit, sc. Caesar. II 43, 3 f.: eius acta in urbe ... quo notiora sunt, minus 
die Grofitaten des Pompeius erfahren, auch nicht zu folgern, daf unsere Quelle mit Letzterem sympathisiert wie zweifellos mit Caesar, dessen virtus, constantia, sublimitas, vigor animi, clementia und magnanimitas der Reihe nach gerühmt werden. Vielmehr werden diese Eigenschaften als für Caesar allein charakteristisch hervorgehoben: $\$ 93$ iustius Pompeio Magno tribuatur DCCCXLVI naves piratis ademisse, Caesari proprium et peculiare sit praeter supra dicta clementiae insigne, woraus man schliefen muß, daß unsere Quelle Pompeius nicht dieselbe virtus $u$. s. w. hat zuerkennen wollen ${ }^{58}$ ). - Das nächste Urteil über die Persönlichkeit des Pompeius begegnet VIII 21, wo sein trauriges Ende als gerechte Vergeltung dafür bezeichnet wird, daß er bei den Spielen seines zweiten Konsulates Elefanten mit barbarischer Grausamkeit habe hinmorden lassen: tanto populi dolore ut oblitus imperatoris ac munificentiae honori suo exquisitae flens universus consurgeret dirasque Pompeio quas ille mox luit poenas imprecaretur, während gleich darauf ( $\$ 22)$ Caesar kein Vorwurf daraus gemacht wird, daß er in seinem dritten Konsulat 20 Elefanten gegen 500 Mann zu Fußs und ein zweites Mal ebensoviele mit Türmen und je 60 Kämpfern darauf gegen ebensoviel Mann Fufvolk wie zuvor und eine gleiche Schar Reiter auftreten ließ. Hier hat auch Münzer (Beitr. 374) die ungünstige Darstellung des P. nicht verkannt. Dieselbe tendenziöse Beurteilung der beiden Männer aber findet sich XVII 243 subsedit in Cumano arbor gravi ostento paulo ante Pompei Magni bella civilia paucis ramis eminentibus . . (244) . . simili modo Trallibus palma in basi Caesaris dictatoris circa bella civilia eius. - Die Nachricht XXXIII 14 ferner, daß unter Pompeius' alleinigem Konsulat 2000 Pfund Gold aus dem Kapitol verschwunden seien, ist "sicher eine Parteianekdote der Caesarianer; die entgegenstehende der Pompeianer, daß Caesar 695 in seinem ersten Konsulat 3000 Pfund Gold aus dem Kapitol gestohlen und durch vergoldetes Kupfer ersetzt habe, giebt

egent stilo. II 46, 1 cum deinde immanes res vix multis voluminibus explicandas C. Caesar in Gallia gereret.

${ }^{58)}$ Vgl. Vell. II 29, 4. 30, 2. 33, 2. 34, 2. 46, 2. 47, 3, dagegen Appian II 150. III, 1 .

Philologus LXY (N. F. XIX), 3. 
Sueton " (Mommsen, Gesch. d. röm. Münzwesens 401 A. 106). Im XXXVII. Buche $\$ 14$ endlich wird das berühmte os probum des Pompeius ganz offenbar an den Pranger gestellt: erat et imago Cn. Pompei e margaritis, illa relicino honore grata, illius probi oris venerandique per cunctas gentes, illa ex margaritis, severitate victa et veriore luxuriae triumpho, numquam profecto inter illos viros durasset cognomen Magni, si prima victoria sic triumphasset! e margaritis, Magne, tam prodiga re et feminis reperta. Wenn Münzer auch diese Nachricht für Pompeius' Parteigänger Varro in Anspruch zu nehmen vermag (Beitr. 284), so scheint sich der Gelehrte hier, seiner Varrohypothese zu Liebe, kaum noch die nötige Unbefangenheit und Besonnenheit bewahrt zu haben. Zu den angeführten Notizen ist noch zu vergleichen XXXIV 200 über berüchtigte Freigelassene des Sulla, Cn. Pompeius, S. Pompeius und anderer aristokratisch gesinnter Männer aus der Zeit der Republik. -

Aehnlich mikgünstig ist die Beurteilung des Sextus Pompeius in der Nat. hist.: X 45 saginare (pavonem) primus instituit circa novissimum piraticum bellum M. Aufidius Lurco, exque eo quaestu redituus HS. sexagena milia habuit, XVI 7 civicae coronae .. cedunt his murales vallaresque et aureae.. cedunt et rostratae ... M. Varrone e piraticis bellis dante Magno Pompeio, itemque M. Agrippa tribuente Caesare e Siculis quae et ipsa piratica fuere. Man ist allgemein geneigt, nach dem Vorgange von Mercklin (de Varrone coronarum interprete praecipuo quaestiones. Dorpat 1859) die Beschreibung der Eichenkränze im XVI. Buche $\$ 7 \mathrm{ff}$. auf die Autorität Varros zurückzuführen. Schwerlich aber wird der langjährige Parteigänger und Freund des Pompeius den Seekrieg gegen dessen Sohn, der für das Erbe seines großen Vaters eintrat, als ein bellum piraticum bezeichnet haben. In der zur Zeit des Seekrieges verfäiten und im Jahre der Entscheidungsschlacht veröffentlichten Schrift de re rustica wird nicht einmal des durch die Unterbrechung der Kornzufuhr aus Sicilien in Rom hervorgerufenen Getreidemangels gedacht, ja gar nicht mehr mit sicilischem Getreide gerechnet, sondern es werden ausdrücklich nur Africa und Sardinien als Korn- 
kammern Roms genannt (r. r. II praef. 4). Ist nicht der Schlufis ex silentio gestattet, daf sich der alte Varro dem Seekrieg gegenüber völlig gleichgültig verhalten, wenn nicht mit seinem Herzen auf Seiten des S. Pompeius gestanden hat, mit dem ein grobier Teil der Bevölkerung Roms sympathisierte (Gardthausen, Augustus I 260, 271) und dem selbst seine Gegner eine gewisse Größse nicht haben absprechen können? Es werden also die beiden Nachrichten des X. und XVI. Buches für einen Schriftsteller in Anspruch zu nehmen sein, welcher seine politischen Anschauungen im Zeitalter des $\mathrm{Au}^{-}$ gustus gebildet hat, der bekanntlich nicht anstelt, in seinem Rechenschaftsbericht den Krieg gegen Sextus als einen Seeräuber- und Sklavenkrieg zu bezeichnen (Mon. Anc. 25 mare pacavi a praedonibus, eo bello servorum. Vgl. Mommsen, RGDA p. 97 sq. und n. h. XXXIV 200 s. o.). Zu vergleichen ist ferner VII 178 eine Parteianekdote der Pompeianer als ingens exemplum lügenhafter Prophezeiung und IX 55 eine Weissagung an Octavian die Ueberwindung des Sextus betreffend, neben der oben angeführten offenbar tendenziösen Stelle im XXXIV. Buche die beiden einzigen in der Nat. hist., an denen der Name des Sextus Pompeius genannt wird. -

Recapitulieren wir das Ergebnis unserer auf der inneren Kritik der Berichte beruhenden Untersuchung, so verliert schon hiedurch allein, abgesehen von allen anderen Indicien, die Annahme einer starken Ausnutzung Varronischer Schriften sehr an Wahrscheinlichkeit ${ }^{5}$ ).

\section{5.}

Um nun einen terminus ante quem für die Hauptquellen der Nat. hist. zu gewinnen, gehe ich aus von der auch schon

59) Vgl. m. Diss. S. 62 A. 28. Selbst die uns erhaltene landwirtschaftliche Schrift scheint Plinius nur durch Vermittelung eines Antiquars der ersten Kaiserzeit gekannt zu haben. Vgl. r. r. III 6, 1 de pavonibus nostra memoria greges haberi coepto et venire magno, ex iis M. Aufidius Lurco supra sexagena milia nummum in anno dicitur capere mit $\mathrm{n}$. h. $\mathrm{X} 45$ s. o. und $\mathrm{m}$. Diss. S. $35 \mathrm{zu} \mathrm{n}$. h. VII 211 . Die hier ausgesprochene Vermutung, daß Pl.' Quelle die Inschrift des Tempels von Ardea unabhängig von Varro gekannt habe, wird durch die Beschreibung des Tempels n. h. XXXV 17 und 115 (aus Verrius, dem die $\$ \S 19$ ff. verdankt werden. Vgl. m. Diss. S. $29 \mathrm{f}$.) bestätigt. 
von anderen gemachten Beobachtung, dass encyclopädische und fachwissenschaftliche Werke wie Strabo, Celsus, Valerius Maximus, Mela, Cornelius Valerianus, Senecas naturales quaestiones, Columella, Masurius Sabinus' Memorialien, Dioscorides u. a. von Plinius seltsamerweise gar nicht oder nur als Zusatzquellen benutzt worden sind. Dieser Schwierigkeit hat man durch die Annahme einer besonderen Vorliebe unseres Autors für die "Antiquitas" entgehen zu können geglaubt ${ }^{60}$ ). Dieselbe eigenartige Vorliebe für die alte Zeit aber wurde früher auch dem Periegeten Pausanias zugeschrieben, wenn er aus der älteren Zeit sogar Unbedeutendes erwähnt, aus der späteren hingegen bedeutsame Tatsachen mit Stillschweigen übergeht, bis man zu der Erkenntnis gelangte, daß Pausanias „eine alte Vorlage gedankenlos ausschreibt* (v. Wilamowitz, Hermes XII S. 346). Lassen sich nun obenein in der Nat. hist. Stellen genug nachweisen, an denen Plinius Tatsachen seiner Zeit unerwähnt läfit, die im Zusammenhang von einem selbständig arbeitenden Schriftsteller nicht leicht hätten übergangen werden können, also die Abhängigkeit unseres Autors von einer älteren Quelle bekunden ${ }^{61}$ ), so liegt die Vermutung nahe, daßs die Hauptquellen der Nat. hist. vor des Verfassers eigener $d$. h. der claudisch-neronischen Zeit und vor den oben genannten Schriftstellern, mithin in der augustisch-tiberischen Zeit bis etwa zum Jahre 25 oder 26 unserer Zeitrechnung geschrieben haben ${ }^{62}$ ).

$\left.{ }^{60}\right)$ Peter Wochenschr. f. class. Philol. $1898 \mathrm{Sp} .71 .74$.

${ }^{61}$ Vgl. m. Diss. S. 15 A. S. 47 A. S. 50 A. S. 51 A. 3.

${ }^{62}$ V Vl. über die Hauptquelle der Bücher III-VI Schweder Phil. LIV S. 535: 'Man hat auch den Umstand als bemerkenswert hervorgehoben, daß Pl. die Geographie des Strabo nicht gekannt hat, und Païs (Straboniana p. 237) schließt daraus, daß Strabo sein Werk fern von Rom zum Abschluß gebracht habe. Zu solchem Trugschluß muß derjenige freilich kommen, dem die wahre Quelle der Plinianischen Geographie unbekannt bleibt. Plinius citiert seine griechischen Gewährsmänner fast ausnahmslos durch Vermittelung einer römischen Schrift von außerordentlich reichem Inhalt, und wenn er den Strabo nicht nennt, so läßt dies schließen, daß Strabo in der Quelle des Plinius nicht citiert war. Wegen der großen Gelehrsamkeit und Litteraturkenntnis, die wir dem unbekannten Verfasser der römischen Quelle des Plinius zuschreiben müssen, ist aber aus der Nichterwähnung des Strabo weiter zu schließen, daß die Quelle des Plinius früher herausgegeben ist als die Geographie des Strabo.' 
Diese mutmaßliche Festlegung des terminus ante quem wird nicht nur durch die auffallende Tatsache bestätigt, daß3 die für die Zeit des Plinius nicht passenden Zeitangaben in der Nat. hist. regelmäßig Schriftquellen der augustisch-tiberischen Zeit indicieren, sondern auch dadurch, daf Antonius in der Nat. hist. seltsamerweise eine ebenso ungünstige wie Tiberius günstige Beurteilung findet. Hinsichtlich der Fälle gedankenloser Herübernahme von Zeitbestimmungen darf auf die $\mathrm{Zu}^{-}$ sammenstellung in meiner Dissertation verwiesen werden. Was aber die nur auf Schriftsteller der augustisch-tiberischen Zeit passende Beurteilung des Antonius und Tiberius betrifft, wird es angemessen sein, zunächst die in Betracht kommenden Stellen kurz mitzuteilen.

Antonius wird fast überall in der Nat. hist., wo wir ihm begegnen, mit Schmähungen bedacht. So lesen wir VII 56 Toranius mango ... detecta fraude a furente increpitus Antonio ... tempestivam administrationem intulit, ut ille proscriptor animus modo et contumelia furens non aliud in censu magis ex fortuna sua duceret, VII 117 am Schlufs eines Enkomions auf Cicero: tu M. Antonium proscripsisti, VII 134: er habe Fidustius proscribiert quia proscriptus fuisset, VII 147 pessimi cives (d. s. Antonius und Lepidus), VIII 55: er sei mit einer Schauspielerin auf einem mit Löwen bespannten Wagen durch die Straßen von Rom gefahren non sine ostento quodam temporum, generosos spiritus iugum subire illo prodigio significante. nam quod ita vectus est cum mima Cytheride, super monstra etiam illarum calamitatium fuit, IX $119 \mathrm{ff}$.: er habe sich in Aegypten täglich mit auserlesenen Speisen "gemästet", seine Ueppigkeit aber sei von dem Sohne eines Schauspielers noch überboten worden (ne triumviratu suo nimis superbiat Antonius paene histrioni comparatus), XIV $147 \mathrm{f}$. nimirum hanc gloriam (sc. ebrietatis) auferre Cicero (sc. filius) voluit interfectori patris sui M. Antonio. is enim ante eum avidissime adprenderat hanc palmam edito etiam volumine de sua ebrietate, quo patrocinari sibi ausus adprobavit plane, ut equidem arbitror, quanta mala per temulentiam terrarum orbi intulisset. exiguo tempore ante proelium Actiacum id volumen evomuit, quo facile intellegatur ebrius iam 
sanguine civium et tanto magis eum sitiens, XXXIII 50: er habe das edelste Metall für die alltäglichsten Bedürfnisse in Gebrauch genommen (Antonius solus contumelia naturae vilitatem auro fecit, o dignum proscriptione, sed Spartaci), XXXIII 83 von dem Raub einer Götterstatue im Partherfeldzug, XXXIII 132 von der Falschmünzerei des Antonius, XXXIV 6 non alia de causa Verrem quem M. Cicero damnaverat proscriptum cum eo ab Antonio quoniam Corinthiis (aeribus) cessurum se ei negavisset, XXXIV 58 von dem Raub einer Götterstatue (Apollinem quem ab triumviro Antonio sublatum restituit Ephesiis divus Augustus admonitus in quiete ${ }^{63}$ )), XXXIV 200 von 'sanguine Quiritium et proscriptionum licentia' berüchtigten Freigelassenen, deren Schande auf ihre Herren zurückfällt, unter denen neben Sulla, Q. Catulus, L. Lucullus, Cn. Pompeius, S. Pompeius, die in den officiösen Darstellungen der augustisch-tiberischen Zeit ungünstig beurteilt werden, auch Antonius genannt wird.

Diesen durch die Heftigkeit ihrer Invectiven frappierenden Nachrichten gegenüber, die im Munde des den Zeitverhältnissen fremd und fern gegenüberstehenden Verfassers der Nat. hist. befremden müssen, findet sich keine einzige Notiz, worin Rühmenswertes von Antonius berichtet würde. Die offenbar tendenziösen Nachrichten aber sind um so auffallender, als von Antonius sonst nur noch an fünf Stellen der Nat. hist. die Rede ist: X 110, XIX 22, XXI 12, XXXI 11, XXXV 3.

Umgekehrt erscheinen Persönlichkeit und Herrschertätigkeit des Tiberius in der Nat. hist. in einem merkwürdig günstigen Lichte, von wenigen Stellen abgesehen, die nachweislich nicht auf Rechnung der von Plinius benutzten Hauptschriftsteller, sondern des Verfassers selbst oder einer Zusatzquelle zu setzen sind.

So wird VII 84 die Fahrt des Tib. zu seinem kranken Bruder unter den Beispielen hervorragender Geschwindigkeit aufgeführt: cuius rei (der Geschwindigkeit der beiden vor-

89) Vgl. RGDA c. 24 p. $96 \mathrm{M}^{2}$ : in templis omnium civitatium provinciae Asiae victor ornamenta reposui quae spoliatis templis is cum quo bellum gesseram, privatim possederat. 
hergenannten Läufer) admiratio ita demum solida perveniet, si quis cogitet nocte ac die longissimum iter vehiculis Tib. Neronem emensum festinantem ad Drusum fratrem aegrotum in Germaniam, VII 149 seine Entfernung nach Rhodos als eine Schmach für Augustus (contumeliosus privigni Neronis secessus) bezeichnet und $\S 150$ bemerkt, Letzterer sei im besten Einvernehmen mit Livia und Tib. gestorben (uxoris et Tiberi cogitationes, suprema eius cura). Seine Gerechtigkeit ferner wird gerühmt, wenn es von ihm heißt, er habe den eigenen Sohn wegen seiner Schwelgerei mit einem öffentlichen Verweise bestraft (XIX 137) und ein im Nachlaß des Statthalters von Aegypten gefundenes obsianisches Bildnis des Menelaos den Heliopoliten für ihre frommen Gebräuche zurückgeschickt (XXXVI 197). Auch die Beseitigung des Standes der Druiden ${ }^{64}$ ) wird ausdrücklich seinem anstatt wie sonst überall Clandius' Principat zum Ruhme angerechnet (XXX 13. Vgl. m. Diss. S. $15 \mathrm{~A}$.), elenso die Konstituierung des Ritterstandes und die förmliche Festsetzung des Vorrechtes der Ringe (XXXIII 32. Vgl. m. Diss. S. 28). Andere Nachrichten kennzeichnen die Kunstliebe des Kaisers: XXXIV 62 destringentem se quem M. Agrippa ante Thermas suas dicavit mire gratum Tiberio principi, XXXV 28 posuit (scil. tabulas) et Tiberius Caesar .. in templo ipsius Augusti, XXXV 70 archigallum quam picturam amavit Tiberius princeps und XXXV 131 Tiberius Caesar in templo eius (des divus Augustus) dicavit hanc tabulam. Sogar zwei witzige Bemerkungen von ihm werden uns mitgeteilt: praef. 25 über Apion quem Tiberius Caesar cymbalum mundi vocabat quum propriae famae tympanum potius videri posset, die andere XIX 145 über den Spargel.

Den angeführten Nachrichten gegenüber verschwinden diejenigen Notizen, die ein absprechendes Urteil über Tiberius fällen. Von diesen wenigen Stellen aber lässt sich zeigen, dak sie nicht in den Hauptquellen gestanden haben, sondern von unserem Autor zum Ausdruck seines persönlichen Urteils über den Charakter des Kaisers oder aus einer Zusatzquelle hinzugefügt worden sind.

e4) Vgl. neuerdings Ihm Realencycl. V 2. 1734 und H. d'Arbois de Jubainville, Les Druides et les dieux celtiques Paris 1906. 
Die erste derartige Notiz begegnet VII 129: non hominis fuit tam, Hercules, quam libidinis, non formae, Paezontem e spadonibus Seiani III $\lceil$ D $j$ mercante Clutorio Prisco, quam quidem iniuriam lucri fecit ille mercatus in luctu civitatis, quoniam arguere nulli vacabat. Der aus Tacitus (Ann. III 49 ff.) bekannte Clutorius Priscus wurde im Jahre $21 \mathrm{n}$. Chr. ermordet. Also ist der Schlufsatz nicht Eigentum der Quelle, sondern tendenziöser Zusatz des Plinius, der jenen schimpflichen Kauf mit den traurigen Zeiten des damaligen Regimentes in Verbindung bringen wollte, ungeachtet der näheren Zeitverhältnisse, ob der Kauf in der ersten oder zweiten, berüchtigten Regierungsperiode des Tiberius abgeschlossen wurde ${ }^{65}$ ). - An einer anderen Stelle XIV $144 \mathrm{f}$. heift es von Tiberius, er sei in seinem Alter finster und grausam, in seiner Jugend ein Trinker gewesen, auch habe er den L. Piso wegen seiner Trunksucht zum Stadtpräfekten gemacht. Tiberius' Sohn Drusus aber wird als in keiner anderen Hinsicht dem Vater so vollkommen ähnlich bezeichnet. Daß Plinius diese Bemerkungen nicht seiner Hauptquelle verdankt. geht daraus hervor, daß sie die eingehend wiedergegebene Erzählung von der Trinkfestigkeit des Novellius Torquatus ganz ungehörig unterbrechen. Wir lesen nämlich unmittelbar vor den angeführten Notizen: apud nos cognomen etiam Novellius Torquatus .. tribus congiis .. epotis uno impetu, spectante miraculi gratia Tiberio principe, und dieser Bericht wird erst $\S 146$ wieder aufgenommen: Torquato rara gloria non labasse sermone. Was Plinius zu seiner ungeschickten Interpolation veranlaßte, war augenscheinlich die Verwunderung über die Mitteilung seiner Quelle, der ihm selbst nur als finster und grausam (in senecta iam severo atque etiam saevo)

$\left.{ }^{65}\right) \mathrm{Da}$ die Erbitterung unseres Autors über Clutorius' Schandtat weniger moralisch als tendenziös, gegen die Regierung des Tiberius gerichtet (vgl. Gercke Senecastudien S. 168 f. Peter d. gesch. Litt. I 318 f. Detlefsen Untersuch. S. 9. 11), zu verstehen ist, lehren die Bekenntnisse aus seiner eigenen Zeit: VII 34 Hermaphroditos oli m . . in prodigiis habitos, nunc vero in deliciis (vgl. Martial epigr. III 72) und VII 184 nostra aetas adnotavit duos equestris ordinis in eodem pantomimo Mystico tam forma praecellente (mortuos). Daher halte ich es für unstatthaft, wegen des Schlußsatzes unserer Nachricht einen zweiten Clutorius Priscus aus der letzten Regierungsperiode des Tiberius (Prosop. imp. Rom. 1 p. 425 nr. 951) anzunehmen. 
bekannte Tiberius habe dem Schauspiel des berühmten Zechers miraculi gratia beigewohnt ${ }^{66}$ ). Er glaubt, des Kaisers Interesse vielmehr auf seine frühere Neigung zum Trunke zurückführen zu können, bei welcher Gelegenheit er auch von der Trunksucht seines Sohnes und eines seiner Beamten (dessen Tüchtigkeit von Seneca ep. 83.14 gerühmt wird) berichtet. Aehnlich liegt das Verhältnis XXVIII 23 cur sternuentis salutamus, quod etiam Tiberium Caesarem, tristissimum, ut constat, hominum, in vehiculo exegisse tradunt und XXXV 28 posuit (tabulas) et Tiberius Caesar minime comis imperator in templo ipsius Augusti, wo sich die Appositionen durch ihren Gegensatz zu den mitgeteilten Notizen als Interpolationen unseres Autors verraten, der es nicht unterlassen wollte, seine Verwunderung über Nachrichten auszusprechen, die zu dem ihm selbst und seinen Zeitgenossen vorschwebenden Bilde von Tiberius' Charakter nicht zu passen schienen. - Ausser an den angeführten Stellen wird nur noch XXXV 62 gelegentlich einer die Kunstliebe des Fürsten kennzeichnenden Notiz ungünstig über Tiberius geurteilt: (Lysippus fecit) destringentum se quem M. Agrippa ante thermas suas dicavit mire gratum Tiberio principi, non quivit temperare sibi in eo, quamquam imperiosus sui inter initia principatus, transtulitque in cubiculum alio signo substituto, cum quidem tanta p. R. contumacia fuit ut theatri clamoribus reponi apoxyomenon flagitaverit princepsque quamquam adamatum reposuerit. In dieser Nachricht allein könnte schon Plinius' Hauptquelle Tiberius einen Vorwurf daraus haben machen wollen, daf er den Apoxyomenos von den Thermen des Agrippa in seinen Privatbesitz übernahm, zumal Plinius eine ähnliche Notiz zu Anfang desselben Buches XXXV 26 exstat certe eius (M. Agrippae viri rusticitati propioris quam deliciis) oratio magnifica et maxime civium digna de tabulis omnibus signisque publicandis, quod fieri satius fuisset quam in villarum exilia pelli

${ }^{66)}$ Vgl. Tac. Ann. I 76: cur abstinuerit spectaculo ipse, varie trahebant; alii taedio coetus, quidam tristitia ingenii et metu conparationis, quia Augustus comiter interfuisset, non crediderim ad ostentandam saevitiam movendasque populi offensiones concessam filio materiem, quamquam id quoque dictum est. Ann. III 37: nullis voluptatibus avocatus. 
sicherlich seiner Hauptquelle verdankt ${ }^{6 \pi}$ ). Auf jeden Fall aber scheint der oben cursiv gedruckte Concessivsatz von Plinius selbst eingeschaltet $\mathrm{zu}$ sein.

Was folgt nun aus der ebenso gehässigen Darstellung des Antonius wie ehrenvollen Beurteilung des Tiberius für die Quellen der betreffenden Nachrichten?

Daß die plinianischen Notizen über die Proscriptionen des Jahres 43 vor Chr. auf einen Schriftsteller aus einer Zeit zurückgehen müssen, als „Marcus Antonius ein wenig den Sündenbock für die jugendlichen Grausamkeiten des Begründers der Dynastie abgeben mußte ", ist schon von Münzer erkannt worden (Beiträge S. 403 f.). Wenn aber der Gelehrte für jene Nachrichten eine Quelle aus der Claudischen Zeit zu konstruieren versucht hat (a. a. O. S. 401 ff.), so dürfte kaum eine Hypothese weniger glücklich genannt werden. Für die Abfassung gehässiger Antoniusnachrichten wäre nämlich keine Zeit ungeeigneter gewesen als die Regierungszeit des Claudius, eines Tochtersohns des Antonius, von dem bezeugt ist, daß er das Andenken seines Grokvaters nicht minder hochhielt als das seiner übrigen Verwandten und ein Edikt erließ, er wolle den Geburtstag seines Vaters Drusus um so mehr gefeiert wissen, als er auch der seines Großvaters Antonius sei (Suet. Claud. 11). Mit Rücksicht auf die Abstammung des regierenden Kaisers haben daher die Litteraten der claudischen Zeit Antonius günstiger beurteilt als die des augustischtiberischen Zeitalters, wie z. B. Seneca in der an Polybius gerichteten Trostschrift Claudius sprechen läßt: M. Antonius, avus meus, nullo minor nisi eo a quo victus est, tunc cum rem publicam constitueret et triumvirali potestate praeditus nihil super se videret (ad Polyb. 16,1). Da nun auch Caligula und Nero Blutsverwandte des Antonius gewesen sind, bleibt uns keine andere Wahl, als die Quellen der Nat. hist. für die tendenziösen Antoniusnachrichten in der augustischtiberischen Zeit zu suchen ${ }^{68}$ ).

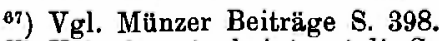

${ }^{68}$ Ueberhaupt scheint erst die Generation der Claudisch-Neronischen Zeit einen gerechten Ausgleich in der Beurteilung des Antonius und Octavian gefunden zu haben. Seneca wenigstens, der in seinen letztęn Schriften 'die Stimmung der im Herzen aristokratisch gesinnten, aber 
Was aber die ehrenvolle Beurteilung von Tiberius' Persönlichkeit und Wirksamkeit in der Nat. hist. betrifft, so wissen wir, daß dieser Kaiser durch das Schreckensregiment in den letzten Jahren seiner Regierung sein Andenken in der Nachwelt derartig verscherzt hatte, daß er in der späteren Litteratur (Seneca, Tacitus, Sueton, Dio) nur noch als grausamer Despot fortlebte, weshalb die Quellen unserer Tiberiusnachrichten unmöglich erst den Zeiten eines Caligula, Claudius oder Nero angehören können.

So schliefen sich Ausgangspunkt und Endpunkt unserer Beweisführung zusammen, daß3 die Hauptquellen der Nat. hist. in der augustisch-tiberischen Zeit bis etwa zum Jahre 25/26 oder höchstens 29 n. Chr., dem Beginn des Schreckensregimentes des Tiberius (Tac. Ann. V 3), gesucht werden müs$\operatorname{sen}^{69}$ ).

Berlin.

M. Rabenthorst.

auch nach Vorurteilslosigkeit strebenden Gebildeten seiner Zeit' (Peter d. geschichtl. Litt. I 461) ausspricht, steht auf der einen Seite nicht an, die Größe des Antonius anzuerkennen (epist. 83, 25: M. Antonium magnum virum et ingenii nobilis) wie er auf der anderen Seite sich nicht scheut, Augustus seine jugendlichen Grausamkeiten vorzuhalten (de ira II 5, 5. III 40, $2 \mathrm{ff}$. Natural. quaest. I 16,1) und gegen ihn sogar mit dem Vorwurf nicht zurückhält, Antonius hinterlistig nach dem Leben getrachtet zu haben (de clem. IX 1), während die officiösen Geschichtsschreiber der augustisch-tiberischen Zeit dasselbe Antonius vorwerfen (Vell. Pat. II 60,5). Vgl. Dirichlet, d. Philosoph Seneca als Quelle, Progr. des Kneiphöfer Gymn. Königsberg 1890.

89) Die Publikation der Verrianischen Rerum memoria dignarum libri, in denen ich die Hauptquelle nicht nur des Plinianischen Werkes, sondern auch der Mela, Columella, Dioscorides, Plutarch, Aetia, Romana, Gellius, Pseudo-Lukian Makrobioi, Solin, Censorin, Macrobius, Isidor, Lydus und eine Hauptquelle vieler anderer (Valerius, Maximus, Seneca nat. quaest., Plutarch de sollertia animalium und Symposiaca, Tacitus Germania, Phlegon, Aelian, Athenaeus, Arnobius, Augustin u. a.) vermute, fällt nachweislich (vgl. m. Diss. S. $58 \mathrm{f}$ ) in die Jahre 23 bis 30 n. Chr. Einen genaueren Termin, wenigstens für gewisse Teile der großen Realencyclopädie, eines ohne Zweifel wahrhaft monströsen Werkes, dessen Umfang darnach ermessen werden mag, da\} schon das Wortlexikon, dessen reiche Materialien und nicht diese allein sich in das Sachlexikon, das letzte Werk des greisen Gelehrten, ergossen, noch in dem Auszug des Festus 20 Bücher umfaßst, wird die Analyse der einzelnen Bücher der Nat. hist. ergeben. 